\title{
A diet high in sugar and fat influences neurotransmitter metabolism and then affects brain function by altering the gut microbiota
}

Yinrui Guo (1)', Xiangxiang Zhu ${ }^{2,3}$, Miao Zeng ${ }^{2,4}$, Longkai Qi ${ }^{2}$, Xiaocui Tang ${ }^{2}$, Dongdong Wang ${ }^{2}$, Mei Zhang ${ }^{4}$, Yizhen $\mathrm{Xie}^{2}$, Hongye $\mathrm{Li}^{3}$, Xin Yang ${ }^{5}$ and Diling Chen $\mathbb{B}^{2}$

\begin{abstract}
Gut microbiota (GM) metabolites can modulate the physiology of the host brain through the gut-brain axis. We wished to discover connections between the GM, neurotransmitters, and brain function using direct and indirect methods. A diet with increased amounts of sugar and fat (high-sugar and high-fat (HSHF) diet) was employed to disturb the host GM. Then, we monitored the effect on pathology, neurotransmitter metabolism, transcription, and brain circularRNAs (circRNAs) profiles in mice. Administration of a HSHF diet-induced dysbacteriosis, damaged the intestinal tract, changed the neurotransmitter metabolism in the intestine and brain, and then caused changes in brain function and circRNA profiles. The GM byproduct trimethylamine-n-oxide could degrade some circRNAs. The basal level of the GM decided the conversion rate of choline to trimethylamine-n-oxide. A change in the abundance of a single bacterial strain could influence neurotransmitter secretion. These findings suggest that a new link between metabolism, brain circRNAs, and GM. Our data could enlarge the "microbiome-transcriptome" linkage library and provide more information on the gut-brain axis. Hence, our findings could provide more information on the interplay between the gut and brain to aid the identification of potential therapeutic markers and mechanistic solutions to complex problems encountered in studies of pathology, toxicology, diet, and nutrition development.
\end{abstract}

\section{Introduction}

Gut microbiota (GM) metabolites can potentially modulate nearly all aspects of host physiology ${ }^{1}$, from regulating immunity ${ }^{2}$ and metabolism ${ }^{3}$ in the gut to shaping mood and behavior ${ }^{4}$. These metabolites can act locally in the intestine or can accumulate up to millimolar concentrations in the serum and organs ${ }^{5}$. Studies have shown that formation of a gut-brain neural circuit for

Correspondence: Xin Yang (37739362@qq.com) or

Diling Chen (diling1983@163.com)

${ }^{1}$ School of Basic Medical Science, Guangzhou University of Chinese Medicine, Guangdong, Guangzhou 510120, China

${ }^{2}$ State Key Laboratory of Applied Microbiology Southern China; Guangdong Provincial Key Laboratory of Microbial Culture Collection and Application; Guangdong Open Laboratory of Applied Microbiology; Institute of

Microbiology, Guangdong Academy of Sciences, Guangzhou 510070, China Full list of author information is available at the end of the article

These authors contributed equally: Yinrui Guo, Xiangxiang Zhu sensory transduction of nutrients enables the gut to inform the brain of all occurrences, and make sense of what has been eaten ${ }^{6}$. Recent studies have revealed that the GM is important in neurodegenerative diseases, including Alzheimer's disease $(\mathrm{AD})^{7,8}$ and Parkinson's disease (PD), and that targeting the GM or/GM metabolites could be used to treat neurodegenerative diseases ${ }^{9,10}$.

An imbalanced diet that includes a high intake of sugar and fat and insufficient dietary fiber over a long time can cause enteric dysbacteriosis. The latter increases the permeability of the intestinal mucosa, and results in abnormalities in intestinal immunity and glucolipid metabolism. At this time, the dominant bacteria can change readily. For example, Fujisaka and colleagues showed that the relative abundance of Bifidobacterium species and Bacillus species decreased in mice fed a highfat diet, whereas the abundance of Gram-negative bacteria

\section{(c) The Author(s) 2021}

(c) (i) Open Access This article is licensed under a Creative Commons Attribution 4.0 International License, which permits use, sharing, adaptation, distribution and reproduction cc) in any medium or format, as long as you give appropriate credit to the original author(s) and the source, provide a link to the Creative Commons license, and indicate if changes were made. The images or other third party material in this article are included in the article's Creative Commons license, unless indicated otherwise in a credit line to the material. If material is not included in the article's Creative Commons license and your intended use is not permitted by statutory regulation or exceeds the permitted use, you will need to obtain permission directly from the copyright holder. To view a copy of this license, visit http://creativecommons.org/licenses/by/4.0/. 
increased $^{11}$. Recent studies have shown that obesity is not necessary for dysfunction of the intestinal barrier. That is, hyperglycemia is more likely to drive intestinal-barrier dysfunction and the risk of enteric infection. Hyperglycemia increases the permeability of the intestinal barrier, which provides microbes with more chances to enter the body and cause proliferation of pathogenic bacteria and focal shifts ${ }^{12}$. Studies have demonstrated that some bacteria can produce bioactive neurotransmitters ${ }^{13,14}$, and these neurotransmitters are thought to regulate the nervous system activity and behavior of the host ${ }^{15,16}$. Recently, O Donnell and coworkers revealed that the neuromodulator tyramine produced by commensal bacteria of Providencia species (which colonize the gut) bypassed the requirement for host tyramine biosynthesis and manipulated a host sensory decision in Caenorhabditis elegans ${ }^{17}$. However, how these bacteria release signals to activate the brain is not known.

Using animal models, several pathways of communication have been identified along the "gut-brain-axis", including those driven by the immune system, vagus nerve, or by modulation of neuroactive compounds by the microbiota $^{18,19}$. In recent years, microbiota have been shown to produce and/or consume a wide range of mammalian neurotransmitters, including dopamine, norepinephrine, serotonin, or gamma-aminobutyric acid ${ }^{13,20}$. Accumulating evidence in animals suggests that manipulation of these neurotransmitters by bacteria may have an impact in host physiology. Preliminary clinical studies have revealed that microbiota-based interventions can also alter neurotransmitter levels ${ }^{13}$. Nonetheless, substantially more work is required to determine if microbiota-mediated manipulation of human neurotransmission has physiological implications and, if so, how it may be exploited therapeutically.

We chose a diet with increased amounts of sugar and fat (i.e., a high-sugar and high-fat (HSHF) diet) to disturb the $\mathrm{GM}$, then monitored the effect on pathology, neurotransmitters, metabolism, and transcription of circularRNAs (circRNAs) in mice. We aimed to identify some associations between the functions of the GM, neurotransmitters, and the brain. We also aimed to enlarge the "microbiome-transcriptome" linkage library. This approach would provide more information on the interplay between the gut and brain to aid identification of potential therapeutic markers and mechanistic solutions to complex problems encountered in studies on pathology, toxicology, diets and nutrition development.

\section{Materials and methods \\ Animals \\ Ethical approval of the study protocol}

All experimental protocols were approved by the Center of Laboratory Animals of the Guangdong Institute of
Microbiology (Guangzhou, China). All efforts were made to minimize the number of animals used.

\section{Preparation and treatments of mice suffering from dysbacteriosis}

Adult male KM mice (18-22 g, 6 weeks) were obtained from the Center of Laboratory Animals of Guangdong Province (certificate number: SCXK [Yue] 2008-0020, SYXK [Yue] 2008-0085). They were pair-housed in plastic cages in a temperature-controlled $\left(25 \pm 2^{\circ} \mathrm{C}\right)$ colony room at a 12-h light-dark cycle. Food and water were available ad libitum.

Mice were allocated randomly into two groups of 12: control and model. The mice in the control group were fed a standard diet. The mice in the model groups were fed a HSHF diet. Water was available freely. These treatments lasted for 3 months.

The components of the HSHF diet were $20 \%$ sucrose, $15 \%$ fat, $1.2 \%$ cholesterol, $0.2 \%$ of bile acid sodium, $10 \%$ casein, $0.6 \%$ calcium hydrogen phosphate, $0.4 \%$ stone powder, $0.4 \%$ premix, and $52.2 \%$ basic feed. Heat ratio: protein $17 \%$, fat $17 \%$, carbohydrate $46 \%$.

\section{Preparation and treatment of SAMP8 mice and newborn KM mice}

Male SAMP8 mice (5 months; mean bodyweight, $20 \pm 5 \mathrm{~g})$ were purchased from Beijing HFK Bioscience (SCXK [Jing] 2014-0004). Adult KM mice (18-22 g, 16 females and 8 males, 8 weeks) were obtained from the Center of Laboratory Animal of Guangdong Province (SCXK [Yue] 2008-0020, SYXK [Yue] 2008-0085). All mice were pair-housed in plastic cages in a temperature-controlled $\left(25 \pm 2{ }^{\circ} \mathrm{C}\right)$ colony room with a 12-h light-dark cycle. Mice had free access to food and water. All animals were allowed to acclimatize to their surroundings for $\geq 1$ week before initiation of experimentation.

\section{Effects of TMA on Sprague-Dawley rats}

Twenty male Sprague-Dawley rats (180-220 g) obtained from the Center of Laboratory Animal of Guangdong Province (SCXK [Yue] 2008-0020, SYXK [Yue] 2008-0085). They were pair-housed in plastic cages in a temperature-controlled $\left(25 \pm 2{ }^{\circ} \mathrm{C}\right)$ colony room at a 12-h light-dark cycle. Food and water were available ad libitum.

Male Sprague-Dawley rats were divided into two groups of 10: normal group, trimethylamine (TMA)induced group $(2 \mathrm{~mL} / \mathrm{kg} / \mathrm{d}$ of $2.5 \%$ TMA purchased from Shanghai Aladdin Biochemical Technology, Shanghai China). 


\section{Influences of Candida albicans and Klebsiella pneumoniae on the cholinergic system in mice}

The abundance of C. albicans (a symbiotic opportunistic pathogen in humans) and K. pneumoniae was found to be high in AD patients in our other study (data not shown). We chose these pathogens to ascertain the potential routes of communication/interaction between the host and its resident bacteria on neurotransmitter metabolism and brain function. $C$. albicans and $K$. pneumoniae were administered (i.g.) to normal C57 mice by monotherapy or in combination.

Adult male C57 mice (18-22 g, 6 weeks) obtained from Center of Laboratory Animal of Guangdong Province, SCXK [Yue] 2008-0020, SYXK [Yue] 2008-0085 were pairhoused in plastic cages in a temperature-controlled $(25 \pm$ $2{ }^{\circ} \mathrm{C}$ ) colony room at a 12 -h light-dark cycle. Twenty C57 mice were divided into four groups: normal, C. albicanstreated, K. pneumoniae-treated, and C. albicans $+K$. pneumoniae-treated $(\mathrm{Ca}+\mathrm{Kp})$.

\section{Measurement of physiological and biochemical indices}

The appearance, behavior, and fur color of animals were documented every day. Bodyweight was measured every 3 days during the period of drug administration. Blood samples were drawn by removing the eye under anesthesia (isoflurane). Serum was acquired by centrifugation and stored at $-80{ }^{\circ} \mathrm{C}$ until measurement. Levels of triglycerides (TG), total cholesterol, (T-CHO) and highdensity lipoprotein-cholesterol (HDL-C) were measured with commercially available kits from Nanjing Jiancheng Bioengineering Institute (Jiangsu, China). Serum and brain-tissue levels of trimethylamine-n-oxide (TMAO), and neurotransmitter levels were quantified using liquid chromatography-mass spectrometry (LC-MS).

\section{Histopathology and immunostaining}

Brain, liver, renal, spinal marrow, spleen, and adipose tissues were removed and fixed in $4 \%$ paraformaldehyde at $\mathrm{pH}$ 7.4 for further pathologic observation. These tissue samples were made into paraffin sections after drawing materials, fixation, washing, dehydration, transparency, dipping in wax, and embedding. Obesityrelated parameters or other related pathologic changes were measured.

The brains of animals were dissected. Four brains from each group were fixed in $4 \%$ paraformaldehyde solution and prepared as paraffin sections. Sections were stained with hematoxylin and eosin (H\&E). silver, or underwent Nissl staining and TUNEL staining. Immunostaining using paraffin-embedded sections (thickness $=3 \mu \mathrm{m}$ ) and a two-step method involving a peroxidaseconjugated polymer kit (Envision ${ }^{\circledR}$; DAKO, Carpinteria, CA, USA) was also done. Slides were observed under light microscopy.

\section{Analyses of microbiome 16S rDNA}

Fresh samples of intestinal content were collected $12 \mathrm{~h}$ before the fasting of rats and stored at $-80^{\circ} \mathrm{C}$. Microbial DNA isolated from these samples, with a total mass ranging from $1.2 \mathrm{ng}$ to $20.0 \mathrm{ng}$, was stored at $-20^{\circ} \mathrm{C}$. Microbial 16S rRNA genes were amplified using a forward primer (5'-ACTCCTACGGGAGGCAGCA-3') and a reverse primer (5'-GGACTACHVGGGTWTCTAAT-3'). Polymerase chain reaction (PCR) amplification was done and the products were purified, quantified, and homogenized to form a sequencing library. The established library was firstly inspected by the library, and the qualified library was sequenced by Illumina HiSeq 2500.

\section{Metabolomics analysis}

Aliquots of each standard solution were mixed to generate a stock standard mixture of $4 \mu \mathrm{g} / \mathrm{mL}$ in $50 \%$ acetonitrile. This $4 \mu \mathrm{g} / \mathrm{mL}$ standard mixture $(100 \mu \mathrm{L})$ was mixed with $50 \mu \mathrm{L}$ of sodium carbonate $(100 \mathrm{mM})$ and $50 \mu \mathrm{L}$ of $2 \%$ benzoyl chloride (BZ) or $2 \%$ benzoyl chloride(phenyl- ${ }^{13} \mathrm{C}_{6}$ ) $\left({ }^{13} \mathrm{C}_{6} \mathrm{BZ}\right)$. The reaction mixture was vortexmixed and diluted to $2400 \mathrm{ng} / \mathrm{mL}$ for BZ derivatives and $50 \mathrm{ng} / \mathrm{mL}$ with $50 \%$ acetonitrile for ${ }^{13} \mathrm{C}_{6} \mathrm{BZ}$ derivatives as the internal standard (IS) stock solution. BZ derivatives were serially diluted to $240,120,60,30,12,3,1.2$, and $0.02 \mathrm{ng} / \mathrm{mL}$. To prepare the standard curve, the standard BZ derivative solutions stated above were mixed isometrically with $50 \mathrm{ng} / \mathrm{mL}$ of IS stock solution to generate calibration levels covering a range of $0.01-1200 \mathrm{ng} / \mathrm{mL}$ for all analytes.

\section{Samples of brain tissue}

Samples of brain tissue $(20 \pm 1 \mathrm{mg})$ were homogenized in a 4-volume (vol/wt) precooled aqueous solution of ascorbic acid $(20 \mathrm{mM})$ with TissueLyser ${ }^{\mathrm{TM}}$ JX-24 (Jingxin, Shanghai, China) beads at $30 \mathrm{~Hz}$ for $3 \mathrm{~min}$. The homogenates were supplemented with 15-volume prechilled acetonitrile $\left(-40{ }^{\circ} \mathrm{C}\right)$ and vortex-mixed for $1 \mathrm{~min}$ before centrifugation at $4{ }^{\circ} \mathrm{C}$ and $14,000 \times \mathrm{g}$ for $15 \mathrm{~min}$. Derivatization was started by addition of $40 \mu \mathrm{L}$ of $2 \% \mathrm{BZ}$ to a mixture of $40 \mu \mathrm{L}$ of supernatant solution and $20 \mu \mathrm{L}$ of sodium carbonate $(100 \mathrm{mM})$. Derivatized samples were mixed with IS stock solution to analyze high concentrations of neurotransmitters by ultra-high pressure-tandem mass spectrometry (UPLC-MS/MS) with an injection volume of $1 \mu \mathrm{L}$. The derivatized sample was diluted 10-fold, and the dilution was mixed isometricallywith ISs to analyze low concentrations of neurotransmitters by UPLC-MS/MS with an injection volume of $5 \mu \mathrm{L}$.

\section{Samples of intestinal content}

Frozen samples of intestinal content $(20 \pm 1 \mathrm{mg})$ were homogenized in 5-volume (vol/wt) of a precooled aqueous solution of ascorbic acid $(20 \mathrm{mM})$. After sonication 
for $3 \mathrm{~min}$, the homogenates were supplemented with 15 -volume prechilled acetonitrile $\left(-40^{\circ} \mathrm{C}\right)$ and vortexmixed for $1 \mathrm{~min}$ before centrifugation at $4{ }^{\circ} \mathrm{C}$ and $14,000 \times \mathrm{g}$ for $15 \mathrm{~min}$. Derivatization was started by addition of $40 \mu \mathrm{L}$ of $2 \% \mathrm{BZ}$ to a mixture of $40 \mu \mathrm{L}$ of supernatant and $20 \mu \mathrm{L}$ of sodium carbonate $(100 \mathrm{mM})$. Derivatized samples were mixed with IS stock solution to analyze low concentrations of neurotransmitters by UPLC-MS/MS with an injection volume of $1 \mu \mathrm{L}$. The derivatized sample was diluted 10-fold, and the dilution was mixed isometrically with ISs to analyze high concentrations of neurotransmitters with UPLC-MS/MS with an injection volume of $10 \mu \mathrm{L}$.

\section{UPLC-MS/MS}

UPLC-MS/MS was done on a Acquity UPLC system (Waters, Milford, MA, USA) coupled to a Triple Quad ${ }^{\mathrm{TM}}$ 5500 tandem mass spectrometer (AB Sciex, Framingham, MA, USA). Each sample or standard mixture was injected onto a UPLC BEH C18 column $(100 \mathrm{~mm} \times 2.1 \mathrm{~mm}$, $1.7 \mu \mathrm{m}$; Waters) at a flow rate of $0.4 \mathrm{~mL} / \mathrm{min}$. The mobile phase consisted of $2 \mathrm{mM}$ ammonium acetate with $0.1 \%$ formic acid in water (A) and acetonitrile (B). Chromatographic separation was conducted by a gradient elution program: $0 \mathrm{~min}, 1 \% \mathrm{~B} ; 0.5 \mathrm{~min}, 1 \% \mathrm{~B} ; 1 \mathrm{~min}, 45 \% \mathrm{~B} ; 4 \mathrm{~min}$, $65 \%$ B; $4.2 \mathrm{~min}, 100 \%$ B; $5.2 \mathrm{~min}, 100 \%$ B; $5.3 \mathrm{~min}, 1 \% \mathrm{~B}$; $7 \mathrm{~min}, 1 \% \mathrm{~B}$. The column temperature was $40^{\circ} \mathrm{C}$.

The analytes eluted from the column were ionized in an electrospray ionization source in positive mode. The conditions were: source temperature, $600^{\circ} \mathrm{C}$; curtain gas, $30 \mathrm{psi}$; ion source gas 1, $50 \mathrm{psi}$; ion source gas 2, $50 \mathrm{psi}$; collision gas, $8 \mathrm{psi}$; ion spray voltage, $5500 \mathrm{~V}$; entrance potential, $10 \mathrm{~V}$; collision cell exit potential, $14 \mathrm{~V}$. Scheduled multiple reaction monitoring (sMRM) was used to acquire data in optimized MRM transition (precursor > product), declustering potential, and collision energy (Table 1). The total scan time per cycle was $0.25 \mathrm{~s}$. The samples and standard-curve samples were analyzed simultaneously. AB Sciex Analyst v1.5.2 (https://sciex. com/products/software/analyst-software) was used to control instruments and acquire data using the default parameters and assist manual inspection to ensure the qualitative and quantitative accuracies of each compound. The peak areas of target compounds were integrated and outputted for quantitative calculation.

\section{Isolation and sequencing of RNA}

Total RNA was isolated using QIAzol ${ }^{\mathrm{TM}}$ (Qiagen, Germany) and miRNeasy ${ }^{\mathrm{TM}}$ kits (Qiagen, Germany), including additional DNase I digestion. Then, ribosomal RNA was removed using the Ribo-Zero ${ }^{\mathrm{TM}}$ Magnetic Gold kit (Qiagen, Germany), and enzymatic degradation of linear RNA was done using Rnase $R$ enzyme. Fragmentation buffer was added to fragments. The first chain of complementary
(c)DNA was synthesized with six-base random hexamers. Then, the buffer, dNTPs, RNase $\mathrm{H}$, and DNA polymerase I were added to synthesize the second chain of cDNA. Purification using the QiaQuick ${ }^{\mathrm{TM}}$ PCR kit and elution using EB buffer after terminal repair, processing of base A, and addition of sequencing joints were carried out. For next-generation sequencing, $0.5 \mu \mathrm{g}$ of ribosomal RNAdepleted RNA was fragmented and primed. Sequencing libraries were constructed using TruSeq ${ }^{\mathrm{TM}}$ RNA Sample Preparation kits (Illumina, San Diego, CA, USA) and were sequenced by Illumina HiSeq $^{\mathrm{TM}} 2500$ flow cells.

\section{Computational analysis of circRNAs}

First, reads were mapped to the latest University of California Santa Cruz transcript set using Bowtie2 v2.1.0 (http://bowtie-bio.sourceforge.net/bowtie2/index.shtml). Gene expression was estimated using RSEM v1.2.15. For analyses of lincRNA expression, we used the transcripts set from Lncipedia (www.lncipedia.org/). Trimmed mean of M-values (TMM) was used to normalize gene expression. Differentially expressed genes were identified using the edgeR program. Genes showing altered expression with $p<0.05$ and $>1.5$-fold changes were considered to have differential expression. Pathway analysis and network analysis were undertaken using Ingenuity (IPA). IPA computes a score for each network according to the fit of a set of supplied focus genes. These scores indicate the likelihood of focus genes belonging to a network versus those obtained by chance. Score $>2$ indicates a $\leq 99 \%$ confidence that a focus gene network was not generated by chance alone. The canonical pathways generated by IPA are the most significant for the uploaded dataset. Fischer's exact test with a false discovery rate (FDR) option was used to calculate the significance of the canonical pathway.

For analyses of circRNA expression, reads were to mapped a genome using STAR. DCC was employed to identify and estimate expression of a particular circRNA. TMM was used to normalize gene expression. Differentially expressed genes were identified using edgeR. miRanda was employed to predict the miRNA target of the circRNA. R (R Institute for Statistical Computing, Vienna, Austria) was used to generate figures.

\section{circRNA verification by real-time reverse transcription- quantitative polymerase chain reaction (RT-qPCR)}

We wished to validate the reliability of high-throughput RNA-sequencing (RNA-seq) and to explore expression of circRNAs during aging. Hence, expression of circRNAs was measured by RT-qPCR. With reference to the method described by Memczak, two sets of primers for each circRNA were designed using Primer Express v5.0 (Table 2): an outward-facing set to amplify only the circRNA, and an opposite-directed set to amplify the linear form. 
Table 1 Different expression of circRNAs in the brain of high sugar \& fat diet induced dysbacteriosis mice (control vs model group, |Foldchange| $>1.50, p<0.05)$.

\begin{tabular}{|c|c|c|c|c|}
\hline circRNA_ID & Gene & mmu_circbase_id & Foldchange & $P$ Value \\
\hline chr12_38147502_38190134_+ & Dgkb & NA & -404.091 & $3.28 \mathrm{E}-06$ \\
\hline chr10_90914692_90948672_+ & Anks1b & mmu_circ_0002557 & -401.022 & $4.29 \mathrm{E}-06$ \\
\hline chr17_44638512_44639826_- & Runx2 & mmu_circ_0006773 & -356.377 & $5.55 \mathrm{E}-05$ \\
\hline chr14_14745967_14757431_+ & Slc4a7 & NA & -244.951 & 0.00029 \\
\hline chr13_83625264_83635469_+ & Mef2c & NA & -243.943 & 0.000387 \\
\hline chr14_21833800_21838043_+ & Vdac2 & NA & -227.906 & 0.000523 \\
\hline chr8_70251613_70252101_+ & Sugp2 & mmu_circ_0014931 & -221.4 & 0.000599 \\
\hline chr1_155187777_155197435_+ & Stx6 & mmu_circ_0008281 & -220.455 & 0.001103 \\
\hline chr4_106420433_106423562_+ & Usp24 & NA & -213.924 & 0.000763 \\
\hline chr6_110914326_110915171_+ & Grm7 & mmu_circ_0012931 & -201.04 & 0.001351 \\
\hline chr1_156869440_156888030_- & Ralgps2 & mmu_circ_0008300 & -185.795 & 0.001516 \\
\hline chr17_84710290_84716715_- & Lrpprc & NA & -181.382 & 0.002065 \\
\hline chr10_5172887_5175916_- & Syne1 & NA & -180.507 & 0.001669 \\
\hline chr5_23565113_23629477_- & Srpk2 & NA & -179.396 & 0.001811 \\
\hline chr8_85875633_85957654_+ & Phkb & mmu_circ_0015077 & -174.144 & 0.001996 \\
\hline chr4_88196758_88197016_+ & Focad & mmu_circ_0011840 & -172.585 & 0.004101 \\
\hline chr19_5797448_5798075_- & Malat1 & NA & -171.865 & 0.002093 \\
\hline chr7_63615288_63685551_+ & Otud7a & NA & -168.418 & 0.00227 \\
\hline chr4_86249762_86252840_+ & Adamtsl1 & mmu_circ_0011813 & -168.018 & 0.002479 \\
\hline chr9_10419035_10658287_- & Cntn5 & NA & -167.399 & 0.002821 \\
\hline chr11_36273230_36300430_- & Tenm2 & mmu_circ_0003007 & -166.71 & 0.003624 \\
\hline chr9_60649479_60680171_- & Lrrc49 & NA & -164.043 & 0.003149 \\
\hline chr14_32250016_32262831_+ & Parg & mmu_circ_0000520 & -160.076 & 0.002826 \\
\hline chr7_126169128_126174248_- & Xpo6 & mmu_circ_0013871 & -159.96 & 0.003022 \\
\hline chr13_97929410_97936861_- & Arhgef28 & mmu_circ_0004930 & -157.107 & 0.003519 \\
\hline chr5_106618070_106666845_- & Zfp644 & mmu_circ_0001380 & -152.411 & 0.003532 \\
\hline chr5_137325322_137325674_- & Slc12a9 & NA & -149.073 & 0.005074 \\
\hline chr8_94047271_94055344_+ & Ogfod1 & NA & -145.577 & 0.006017 \\
\hline chr9_21934117_21935628_+ & Ccdc159 & NA & -145.057 & 0.005719 \\
\hline chr6_101169133_101172447_- & Pdzrn3 & mmu_circ_0012894 & -144.036 & 0.004294 \\
\hline chr13_8842729_8861265_- & Wdr37 & NA & -141.159 & 0.004877 \\
\hline chr10_58467114_58470762_+ & Ranbp2 & NA & -140.656 & 0.004984 \\
\hline chr12_3865984_3873428_+ & Dnmt3a & mmu_circ_0003954 & -139.642 & 0.004835 \\
\hline chr6_127729409_127732820_- & Prmt8 & NA & -137.936 & 0.004863 \\
\hline chr8_106981125_106981339_+ & Sntb2 & mmu_circ_0001722 & -136.429 & 0.00534 \\
\hline chr12_111097454_111100038_+ & Rcor1 & mmu_circ_0003755 & -135.001 & 0.005934 \\
\hline chr8_99400725_99401177_- & Cdh8 & NA & -133.903 & 0.005448 \\
\hline chr18_86461041_86473843_+ & Neto1 & NA & -133.846 & 0.005985 \\
\hline chr13_76822972_76925525_+ & Mctp1 & NA & -133.839 & 0.006304 \\
\hline
\end{tabular}


Table 1 continued

\begin{tabular}{|c|c|c|c|c|}
\hline circRNA_ID & Gene & mmu_circbase_id & Foldchange & $P$ Value \\
\hline chr9_16021315_16031420_- & Fat3 & mmu_circ_0015421 & -131.089 & 0.006281 \\
\hline chr7_97031337_97040036_+ & Nars2 & NA & -130.142 & 0.005893 \\
\hline chr15_79542486_79543856_- & $\mathrm{Ddx} \times 17$ & NA & -129.349 & 0.005771 \\
\hline chr10_119986331_120010286_+ & Grip1 & NA & -128.055 & 0.005706 \\
\hline chr5_141962664_141975714_+ & Sdk1 & mmu_circ_0012262 & -127.064 & 0.007112 \\
\hline chr11_4803677_4816165_- & $\mathrm{Nf} 2$ & NA & -124.657 & 0.006556 \\
\hline chr12_77277360_77365359_+ & Fut8 & mmu_circ_0004207 & -123.76 & 0.007571 \\
\hline chr13_30817997_30826094_- & Exoc2 & NA & -122.612 & 0.007436 \\
\hline chr3_55477804_55489896_+ & Dclk1 & mmu_circ_0010690 & -122.425 & 0.006334 \\
\hline chr9_114705053_114709314_+ & Dync1li1 & mmu_circ_0015352 & -119.173 & 0.007756 \\
\hline chr5_140430051_140433134_+ & Eif3b & NA & -117.743 & 0.007017 \\
\hline chr9_86634645_86679952_- & Me1 & NA & -115.342 & 0.007181 \\
\hline chr16_13790620_13796077_+ & $\operatorname{Rrn} 3$ & NA & -115.165 & 0.007354 \\
\hline chr4_109037974_109039914_+ & Nrd1 & NA & -113.931 & 0.007381 \\
\hline chr14_77365025_77441899_+ & Enox1 & NA & -110.46 & 0.008948 \\
\hline chr10_45648590_45649987_+ & Hace1 & NA & -108.435 & 0.008376 \\
\hline chr1_5095614_5135937_+ & Atp6v1h & mmu_circ_0008758 & -107.867 & 0.008232 \\
\hline chr7_97705588_97716539_+ & Clns1a & mmu_circ_0013679 & -104.857 & 0.008756 \\
\hline chr6_36894775_36916461_- & Dgki & NA & -104.678 & 0.00873 \\
\hline chr9_22643744_22659145_+ & Bbs9 & mmu_circ_0015468 & -104.402 & 0.008172 \\
\hline chr4_128296394_128338697_+ & Csmd2 & mmu_circ_0011199 & -103.867 & 0.008444 \\
\hline chr8_123968000_123969922_- & Abcb10 & NA & -103.404 & 0.009222 \\
\hline chr2_69944299_69948510_+ & Ubr3 & NA & -103.071 & 0.009949 \\
\hline chr17_11635309_11673052_+ & Park2 & NA & -102.934 & 0.009596 \\
\hline chr4_36718866_36724085_- & Lingo2 & mmu_circ_0011588 & -102.814 & 0.009541 \\
\hline chr15_38498668_38514609_- & Azin1 & NA & -102.811 & 0.008268 \\
\hline chr4_32826929_32828808_- & Ankrd6 & NA & -101.776 & 0.008501 \\
\hline chrX_140384002_140391454_+ & Frmpd3 & NA & -101.259 & 0.008774 \\
\hline chr1_135394135_135400203_- & Ipo9 & mmu_circ_0008200 & -101.042 & 0.008981 \\
\hline chr4_132656692_132673032_+ & Eya3 & mmu_circ_0001277 & -100.539 & 0.008743 \\
\hline chr18_43562530_43567210_- & Jakmip2 & NA & -100.345 & 0.012209 \\
\hline chr19_27800116_27806908_- & $\mathrm{Rf} \times 3$ & NA & -98.2611 & 0.01054 \\
\hline chr3_86816357_86831834_- & Dclk2 & NA & -95.8757 & 0.009228 \\
\hline chr12_87075077_87076505_+ & Tmem63c & NA & -95.0844 & 0.010211 \\
\hline chr15_12457797_12458299_- & Pdzd2 & mmu_circ_0005537 & -94.292 & 0.009719 \\
\hline chr9_77164764_77181396_- & Mlip & mmu_circ_0015896 & -94.1348 & 0.010842 \\
\hline chr5_124632878_124639283_+ & Atp6v0a2 & NA & -92.7345 & 0.010875 \\
\hline chr2_158473122_158477763_+ & Ralgapb & mmu_circ_0009519 & -91.3784 & 0.010868 \\
\hline chr11_4799860_4820493_- & Nf2 & mmu_circ_0003054 & -90.1746 & 0.009803 \\
\hline
\end{tabular}


Table 1 continued

\begin{tabular}{|c|c|c|c|c|}
\hline circRNA_ID & Gene & mmu_circbase_id & Foldchange & $P$ Value \\
\hline chr18_12871077_12906949_- & Osbpl1a & NA & -89.874 & 0.011312 \\
\hline chr16_56151273_56155375_+ & Senp7 & NA & -89.8398 & 0.010437 \\
\hline chr9_50789496_50792407_+ & Alg9 & NA & -88.074 & 0.011172 \\
\hline chr2_158047918_158058827_+ & Rprd1b & NA & -86.9627 & 0.01007 \\
\hline chr16_38505411_38518532_- & Timmdc1 & NA & -86.7884 & 0.010072 \\
\hline chr6_37048119_37058028_- & Dgki & mmu_circ_0013333 & -86.0778 & 0.010146 \\
\hline chr9_59394877_59412531_- & Arih1 & NA & -85.9027 & 0.00997 \\
\hline chr1_184925815_184945000_- & Mark1 & NA & -85.741 & 0.010481 \\
\hline chr4_58861524_58875573_- & Al314180 & mmu_circ_0011714 & -84.1099 & 0.011083 \\
\hline chr9_24473773_24485101_- & Dpy19|1 & NA & -83.2237 & 0.010312 \\
\hline chr1_143667624_143677855_- & $\mathrm{Cdc} 73$ & mmu_circ_0008242 & -82.7125 & 0.010813 \\
\hline chr8_68460923_68461851_- & Csgalnact1 & mmu_circ_0014912 & -82.7124 & 0.010799 \\
\hline chr6_148411037_148425984_- & Tmtc1 & mmu_circ_0013190 & -81.6378 & 0.010321 \\
\hline chr4_155626773_155628823_+ & Cdk11b & mmu_circ_0011499 & -80.5751 & 0.011413 \\
\hline chr17_87678207_87680078_+ & Msh2 & NA & -80.3893 & 0.010289 \\
\hline chr2_6721416_6747946_- & Celf2 & NA & -80.0149 & 0.012001 \\
\hline chr9_96287600_96310688_+ & Tfdp2 & mmu_circ_0016010 & -78.0619 & 0.012609 \\
\hline chr14_13995006_14053023_+ & Atxn7 & mmu_circ_0005063 & -76.833 & 0.011972 \\
\hline chrX_167363544_167374186_- & Prps2 & NA & -75.4238 & 0.013103 \\
\hline chr4_150431522_150431985_+ & Rere & mmu_circ_0011440 & -65.5894 & 0.012612 \\
\hline chr14_29333708_29396583_- & Cacna2d3 & NA & -65.0899 & 0.013294 \\
\hline chr6_51562017_51589065_+ & Snx 10 & NA & -64.6467 & 0.014026 \\
\hline chr14_62733627_62743998_- & $\operatorname{lnts} 6$ & NA & -64.3073 & 0.01361 \\
\hline chr7_56982624_56985163_- & Gabrg3 & mmu_circ_0014270 & -62.3892 & 0.014665 \\
\hline chr13_91968946_91972285_- & Rasgrf2 & mmu_circ_0004873 & -61.1195 & 0.015346 \\
\hline chr14_79369077_79370097_- & Naa16 & mmu_circ_0005462 & -58.7143 & 0.014048 \\
\hline chr5_35935551_35936132_+ & Afap1 & mmu_circ_0012596 & -58.398 & 0.005375 \\
\hline chr15_25737449_25742449_+ & Myo10 & mmu_circ_0005560 & -58.3059 & 0.014391 \\
\hline chr6_126013159_126015695_+ & Ano2 & NA & -56.5138 & 0.01669 \\
\hline chr7_91449753_91872500_+ & Dlg2 & NA & -55.3916 & 0.016159 \\
\hline chr5_21194399_21207641_+ & Gsap & NA & -54.8773 & 0.01561 \\
\hline chr8_25592451_25594166_- & Letm2 & NA & -54.5229 & 0.01658 \\
\hline chr2_44570418_44591990_- & Gtdc1 & NA & -51.9963 & 0.007337 \\
\hline chr5_128761339_128780376_- & Rimbp2 & mmu_circ_0012149 & -51.8159 & 0.016427 \\
\hline chr13_8672404_8701731_+ & Adarb2 & mmu_circ_0004840 & -51.1778 & 0.007795 \\
\hline chr17_44717191_44724901_- & Runx2 & mmu_circ_0000795 & -50.8107 & 0.012892 \\
\hline chr18_34268297_34300061_+ & Apc & mmu_circ_0007251 & -45.3232 & 0.013193 \\
\hline chr3_118759655_118811267_+ & Dpyd & NA & -44.9853 & 0.018425 \\
\hline chr11_117290397_117291036_+ & 9-Sep & mmu_circ_0002788 & -44.5989 & 0.01282 \\
\hline
\end{tabular}


Table 1 continued

\begin{tabular}{|c|c|c|c|c|}
\hline circRNA_ID & Gene & mmu_circbase_id & Foldchange & $P$ Value \\
\hline chr14_33388799_33402923_- & Mapk8 & NA & -43.5971 & 0.018773 \\
\hline chr9_121364000_121392051_+ & Trak1 & NA & -36.6467 & 0.007726 \\
\hline chr2_80520371_80525678_- & Nckap1 & mmu_circ_0001039 & -33.3722 & 0.010502 \\
\hline chr7_75140075_75149088_- & Sv2b & NA & -29.0699 & 0.002013 \\
\hline chr16_60424720_60425384_- & Epha6 & mmu_circ_0000694 & -29.0005 & 0.01947 \\
\hline chr4_59207760_59213977_+ & Ugcg & NA & -28.8672 & 0.006424 \\
\hline chr17_74770242_74799411_+ & Ttc27 & NA & -23.7796 & 0.018256 \\
\hline chr12_66506105_66506822_- & Mdga2 & mmu_circ_0004128 & -20.9794 & 0.012785 \\
\hline chr7_133862102_133870087_+ & Fank1 & mmu_circ_0013965 & -18.4343 & 0.011266 \\
\hline chr1_164340728_164347879_+ & Nme7 & mmu_circ_0008373 & -18.2923 & 0.005958 \\
\hline chr2_115669581_115687169_+ & BC052040 & mmu_circ_0001057 & -14.198 & 0.016445 \\
\hline chr5_122429485_122433537_+ & Anapc7 & mmu_circ_0012079 & 14.58085 & 0.012689 \\
\hline chr3_125914637_125915461_- & Ugt8a & mmu_circ_0010433 & 14.9599 & 0.014812 \\
\hline chr4_84390888_84414348_- & Bnc2 & mmu_circ_0001224 & 19.49736 & 0.007617 \\
\hline chr5_139181416_139184719_+ & Heatr2 & mmu_circ_0012236 & 56.47568 & 0.006519 \\
\hline chr8_40348939_40359448_+ & Micu3 & NA & 61.38255 & 0.014883 \\
\hline chr8_125886933_125888193_- & Pcnxl2 & NA & 62.23414 & 0.014888 \\
\hline chr17_6198671_6201834_+ & Tulp4 & NA & 64.37413 & 0.003266 \\
\hline chr16_55918537_55923040_- & Cep97 & NA & 71.82017 & 0.013319 \\
\hline chr7_139845584_139852657_+ & Gpr123 & NA & 74.60841 & 0.014702 \\
\hline chr13_59473687_59482604_- & Agtpbp1 & mmu_circ_0004710 & 78.31382 & 0.012486 \\
\hline chr15_64191483_64349913_- & Asap1 & NA & 78.71352 & 0.01227 \\
\hline chr1_89627760_89671271_+ & Agap1 & NA & 79.23549 & 0.014461 \\
\hline chr2_163040626_163042799_+ & Ift52 & NA & 80.06381 & 0.012829 \\
\hline chr4_132910691_132913826_- & Fam76a & NA & 82.11838 & 0.013523 \\
\hline chr16_96152970_96154195_+ & Wrb & NA & 91.7975 & 0.011942 \\
\hline chr17_50782260_50800195_- & Tbc1d5 & NA & 92.09871 & 0.012202 \\
\hline chr8_31849309_31968185_- & $\mathrm{Nrg} 1$ & NA & 92.93369 & 0.012086 \\
\hline chr13_119782771_119790287_- & Zfp131 & NA & 94.26963 & 0.011376 \\
\hline chr5_89038450_89058044_+ & Slc4a4 & NA & 94.35752 & 0.012803 \\
\hline chr7_97636376_97653207_+ & Rsf1 & NA & 95.61444 & 0.010142 \\
\hline chr7_37649685_37658660_- & Zfp536 & NA & 99.19821 & 0.011147 \\
\hline chr6_97189528_97199321_- & Uba3 & mmu_circ_0013630 & 100.1458 & 0.009487 \\
\hline chr19_46449828_46453315_+ & Sufu & NA & 101.4795 & 0.009533 \\
\hline chr5_76265790_76274043_- & Clock & mmu_circ_0012787 & 102.1796 & 0.011921 \\
\hline chr5_34419997_34444913_+ & Fam193a & mmu_circ_0001340 & 103.6374 & 0.009949 \\
\hline chr4_59596862_59601567_+ & $\mathrm{Hsdl2}$ & NA & 105.3806 & 0.010751 \\
\hline chr6_119369859_119370408_- & Adipor2 & mmu_circ_0013007 & 107.8557 & 0.009198 \\
\hline chr10_30762739_30771840_- & Ncoa7 & mmu_circ_0002155 & 108.1671 & 0.008979 \\
\hline
\end{tabular}


Table 1 continued

\begin{tabular}{|c|c|c|c|c|}
\hline circRNA_ID & Gene & mmu_circbase_id & Foldchange & $P$ Value \\
\hline chr8_79070370_79076538_+ & Zfp827 & mmu_circ_0015001 & 111.6757 & 0.008381 \\
\hline chr14_99179314_99196451_+ & Pibf1 & NA & 112.7662 & 0.010379 \\
\hline chr2_10393264_10461472_+ & Sfmbt2 & NA & 114.4586 & 0.008053 \\
\hline chr4_102487210_102515611_+ & Pde4b & NA & 114.7701 & 0.007858 \\
\hline chr16_62769606_62776383_- & Nsun3 & NA & 114.8451 & 0.009533 \\
\hline chr7_126886207_126889060_- & Tmem219 & NA & 115.4738 & 0.008434 \\
\hline chr1_139039999_139110485_+ & Dennd1b & mmu_circ_0000082 & 116.1391 & 0.007941 \\
\hline chr1_156620838_156625583_+ & Abl2 & NA & 116.4107 & 0.007942 \\
\hline chr4_74357341_74377800_+ & $\mathrm{Kdm} 4 \mathrm{c}$ & NA & 116.9301 & 0.007803 \\
\hline chr4_150468694_150470391_+ & Rere & NA & 120.5443 & 0.00708 \\
\hline chr3_152317613_152320743_- & Fam73a & NA & 122.1839 & 0.007135 \\
\hline chr11_102860001_102865660_- & Eftud2 & mmu_circ_0002659 & 122.9623 & 0.008327 \\
\hline chr12_55757925_55762737_- & Ralgapa1 & mmu_circ_0004092 & 124.9689 & 0.006679 \\
\hline chr3_131599812_131607463_+ & Papss1 & NA & 125.3036 & 0.00663 \\
\hline chr13_94210576_94233180_- & Scamp1 & NA & 139.1756 & 0.005192 \\
\hline chr10_49522142_49535499_- & Grik2 & NA & 148.6745 & 0.004614 \\
\hline chr11_23281305_23282726_+ & Xpo1 & mmu_circ_0002894 & 153.5301 & 0.004153 \\
\hline chr5_44592732_44799437_- & Ldb2 & NA & 163.2662 & 0.003521 \\
\hline chr18_82955523_82957449_+ & Zfp516 & mmu_circ_0000909 & 178.6214 & 0.00208 \\
\hline chr8_88156461_88158265_+ & Heatr3 & mmu_circ_0015094 & 451.1435 & 2.85E-06 \\
\hline
\end{tabular}

Table 2 PCR primers.

\begin{tabular}{lll}
\hline Primer name & Sequence $\left(\mathbf{5}^{\prime}\right.$-> $\left.\mathbf{3}^{\prime}\right)$ & Product size (bp) \\
\hline mmu_circ_0012931 & circ_0012931-F1: TCCACTTGTTAAGATACCTC & 168 \\
& circ_0012931-R1: GCAAGAGTAGATACATAATTCC & 100 \\
B-actin & $\beta$-actin-F1: GCTTCTAGGCGGACTGTTAC & \\
& $\beta$-actin-R1: CCATGCCAATGTTGTCTCTT & 179 \\
rno_circ_NF1-419 & mo_circ_NF1-419-F1: AGTCGAATTCTACAAGCTTC & \\
& mo_circ_NF1-419-R2: AGCTTCTCCAAATATCCTCAT & \\
\hline
\end{tabular}

Total RNA was extracted (TRIzol® Reagent, Life Technologies, Carlsbad, CA, USA), digested using RNase $\mathrm{R}$, and purified. cDNA was synthesized using the Geneseed $^{\circledR}$ II First Strand cDNA Synthesis kit (Agilent Technologies, Santa Clara, CA, USA). Outward-facing primers were designed to amplify the fragment across the junction from cDNA, then the fragment was sequenced by Sangon Biological Engineering (Shanghai China). RT-qPCR was undertaken using Geneseed ${ }^{\circledR}$ qPCR SYBR ${ }^{\circledR}$ Green Master Mix (Agilent Technologies). PCR-specific amplification was conducted with an ABI 7500 system (Applied
Biosystems, Foster City, CA, USA). Expression of circRNAs was defined based on the threshold cycle $(\mathrm{Ct})$, and relative expression was calculated via the $2^{-\Delta \Delta \mathrm{Ct}}$ method. Glyceraldehyde 3-phosphate dehydrogenase served as an IS control with all reactions done in triplicate.

\section{Western blotting}

Briefly, global brain tissue was dissected from treated mice (purchased from Beijing HFK Bioscience; SCXK (Jing) 2014-0004) and proteins extracted with a radioimmunoprecipitation assay lysis buffer (T-PER ${ }^{\mathrm{TM}}$ Tissue 
Protein Extraction Reagent; catalog number, 78510; Thermo Scientific, Waltham, MA, USA). Proteins were separated by sodium dodecyl sulfate-polyacrylamide gel electrophoresis and transferred onto polyvinylidene fluoride (PVDF) membranes. After blockade with 5\% nonfat dry milk in Tris-buffered saline $(20 \mathrm{mM}$ Tris- $\mathrm{HCl}, 500 \mathrm{mM}$ $\mathrm{NaCl}, \mathrm{pH} 7.4$ ) with $0.2 \%$ Tween-20 (T104863; Aladdin, Beijing, China), the PVDF membranes were probed with antibodies overnight at $4{ }^{\circ} \mathrm{C}$, followed by incubation with a horseradish peroxidase-conjugated goat anti-mouse (G2211-1-A; Servicebio, Beijing, China) or goat anti-rabbit (G2210-2-A; Servicebio) IgG secondary antibody (1:2000 dilution). Band intensity was quantified using ImageJ (National Institutes of Health, Bethesda, MD, USA).

\section{Statistical analyses}

Data are the mean \pm SD of at least three independent experiments. Significant differences between treatments were analyzed by one-way analysis of variance (ANOVA) at $p<0.05$ using SPSS (IBM, Armonk, NY, USA) and Prism 5 (GraphPad, San Diego, CA, USA).

\section{Results}

\section{The HSHF diet disrupts the GM}

The bodyweights of mice fed a HSHF diet were higher than those fed a standard diet (control, $p<0.05$ ) (Fig. 1A); levels of blood glucose (Fig. 1B) and TMAO (Fig. 1C) were also higher $(p<0.05)$. These data suggested that the HSHF diet utilized caused increases in bodyweight, blood glucose level, and TMAO level. Sequencing of the $16 \mathrm{~S}$ rRNA gene showed that the HSHF diet significantly decreased the operational taxonomic units (OTUs) of bacteria of the phyla Acidobacteria, Verrucomicrobia, Tenericutes, and Firmicutes, while increasing the OTUs of bacteria of the phyla Bacteroidetes, Proteobacteria, Deferribacteres, Cyanobacteria and Actinobacteria $(p<0.05)$ (Fig. 1D and Fig. S1A). The OTUs of bacteria from the genera Bifidobacterium (213.12-fold change compared with that in the control group), Coriobacteriaceae (51.02), Sutterella (18.84), Lactobacillus (14.46), Coprobacillus (3.86), Roseburia (3.56), Odoribaccter (2.76), Dorea (2.65), and Flavobacteriaceae (2.39) were decreased significantly $(p<$ 0.05) (Fig. 2E), whereas those of AF12 (-6.64-fold changes), Bliophila (-13.37), Butyricimonas (-2.14), Paraprevotella (-2.76), Bacteroidales (-2.85), Streptophyta (-20.66), Mucispirillum (-18.50), Candidatus Arthromitus (-5.01), [Mogibacteriaceae] (-4.92), Dehalobacterium (-5.87), RF32 (-4.46), Anaerotruncus (-2.43), Erysipelotrichaceae (-2.73), and Ruminococcaceae $(-2.98)$ were increased significantly $(p<0.05)$ (Fig. 1E and Fig. S1B) following administration of the HSHF diet. The whole GM was different between the two groups of mice: control mice and those fed a HSHF diet. Principal component analysis (Fig. S1C), Venn diagram
(Fig. S1D), and linear discriminant analysis effect size (LEfSe) analysis (Fig. S1E and S1F) could be used to distinguish between these two groups. Hence, the HSHF diet could induce dysbacteriosis in mice. These results are almost identical to data from studies showing that intestinal dysbacteriosis interacts with fat $^{21}$.

\section{The HSHF diet changes the steady state of the intestine}

Intestinal dysbacteriosis were considered first affects the intestinal physiology ${ }^{22}$. The pathology of the small intestine and colon of mice fed the HSHF diet was different from that of mice fed the standard diet. Changes induced by the HSHF diet included: cell shrinkage; reduction of cell turnover; cytoplasmic vacuolar changes; blurred and indistinct cell boundaries; severe shedding of intestinal villi; scattering of damaged tissue blocks (Fig. 1F, G).

Immunohistochemical analyses showed that expression of some proinflammatory markers was affected. Expression of tumor necrosis factor (TNF)- $\alpha$ and nuclear factorkappa B (NF-kB p65) was activated $(p<0.05)$ (Fig. $1 \mathrm{H}-\mathrm{K})$, whereas expression of interleukin (IL) -2 and peroxisome proliferator-activated receptor (PPAR) $-\gamma$ was inhibited $(p<0.05)$ (Fig. 1H-K). RNA-seq of small-intestinal tissues revealed that expression of 42 RNAs was upregulated and that of 68 RNAs was downregulated $(|\log 2 \mathrm{FC}|>1.0$, FDR $<0.05$ vs. control) (Table S1, Fig. S2A). For example, expression of Hspala, Hspa1b, P2ry4, Enpp7, Ano3, Hsph1, Nos1ap, Slc5a12, Slc5a4a, Gm5286, Paqr9, G0s2, Trim50, Lep, Apoa2, Retnlb, Gsdmc, TCONS_00019039, Fam205a4, Gm20708, Exosc6, Pde2a and Gm10184 mRNAs was changed, and these were all related to intestinal injury/leakage, inflammation, and immunity. Pathway analysis using the Kyoto Encyclopedia of Genes and Genomes (KEGG) database showed that the important enrichment pathways were the: "PPAR signaling pathway" (mRNA of APOA2, Acaa1b, Cyp4a10, Fabp1, Hmgcs2, Me1); "biosynthesis of unsaturated fatty acids"; "fructose and mannose metabolism"; "glycolysis/gluconeogenesis"; "fatty acid elongation"; "carbon metabolism"; "renin secretion"; "renin-angiotensin system"; "AMPK signaling pathway" (mRNA of G6pc, Fbp1, Srebf1, Lep). Together these results demonstrated that dysbacteriosis influenced intestinal injury/leakage, the inflammatory response, and energy metabolism. Furthermore, histopathology of the liver (Fig. S2B), kidney (Fig. S2E), spinal marrow (Fig. S2E), spleen (Fig. S2D), adipose (Fig. S2C) and heart (Fig. S2F) tissues showed that the HSHF diet not only induced dysbacteriosis, but also activated inflammation and damage to multiple organs.

\section{The HSHF diet influences the brain cholinergic system and inflammation}

The HSHF diet was administered to mice to disturb the GM. Brain sections from mice were analyzed using 

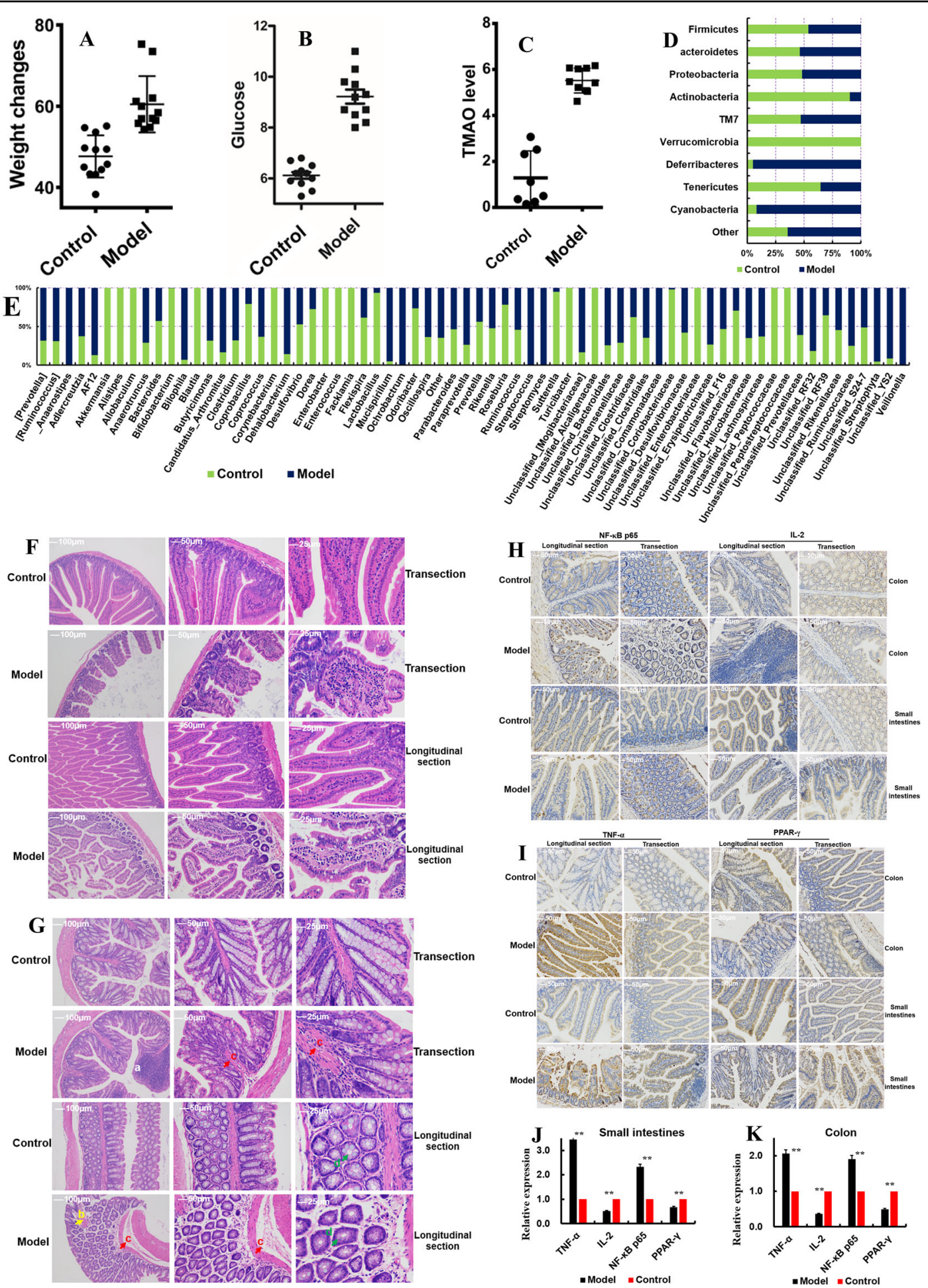

Fig. 1 Dysbacteriosis affects the homeostatic balance of the intestine in mice fed a diet high in sugar and fat for $\mathbf{4}$ months. A Influences of a diet high in sugar and fat on the bodyweight of mice. $\mathbf{B}$ Influences of a diet high in sugar and fat on the blood glucose level of mice. $\mathbf{C}$ Influences of a diet high in sugar and fat on TMAO levels in serum. $\mathbf{D}$ Influences of a diet high in sugar and fat on the gut microbiota at the phylum level. E Influences of a diet high in sugar and fat on the gut microbiota at the genus level, see Fig. S2. F, G Influences of a diet high in sugar and fat on intestine (F) and colon (G) histopathology using H\&E staining, see the pathologic observations in other organs (livers, renal, spinal marrow, spleen, adipose and heart) in Fig. S2. H-K Influences of a diet high in sugar and fat on intestine and colon histopathology using immunohistochemical analyses. Data are the mean \pm SD of more than 8 independent experiments (the histopathology data were the mean \pm SD of more than 3 independent experiments). ${ }^{*} p<0.05$ and ${ }^{* *} p<0.01$ vs. the model group by one-way ANOVA, followed by the Holm-Sidak test. 

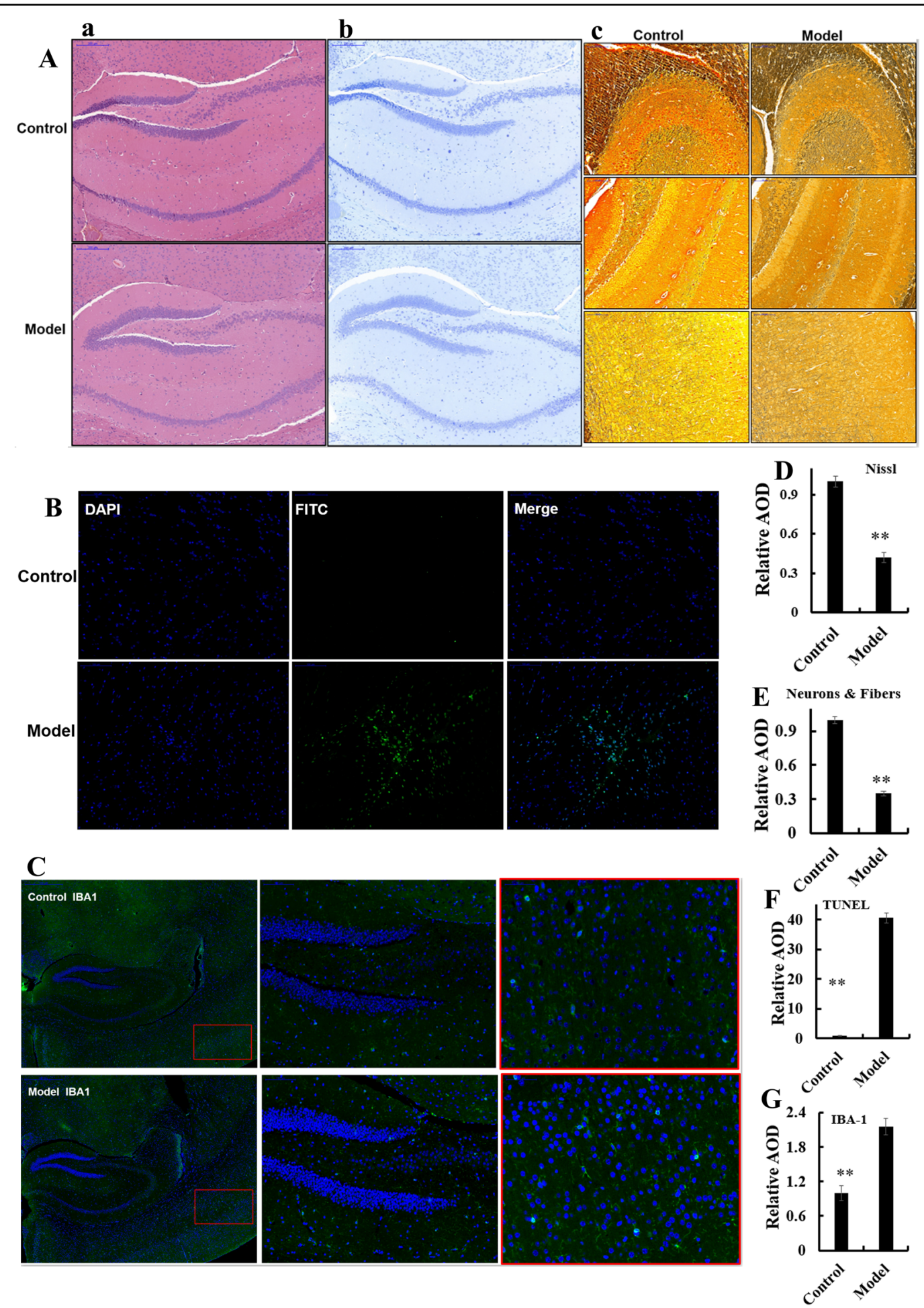

Fig. 2 Dysbacteriosis affects brain histopathology in mice fed a diet high in sugar and fat for $\mathbf{4}$ months. A Samples were stained using hematoxylin and eosin (H\&E), see also Fig. S3A-a. B, D Samples underwent Nissl staining, see also Fig. S31A-b. C, E Samples were stained using silver, see also Fig. S3A-c. D, F Samples underwent TUNEL staining, see also Fig. S3A-d. C, G Samples were stained using immunofluorescent microglial of IBA-1, see also Fig. S3B-b. Data are the mean \pm SD of 5 independent experiments. ${ }^{*} p<0.05$ and ${ }^{* *} p<0.01$ vs. the model group by one-way ANOVA, followed by the Holm-Sidak test. 
various stains. H\&E (Fig. 2Aa, Fig. S3A-A), Nissl (Fig. 2Ab, D and Fig. S3A-B), silver (Fig. 2Ac, E and Fig. S3A-C) and TUNEL (Fig. 2B-F and Fig. S3A-D) staining showed obvious changes in pathology in mice with HSHF diet-induced dysbacteriosis: reduction in the size and turnover of neurons; cytoplasmic vacuolar changes; nerve-fiber reduction. In particular, the apoptotic percentage in the hypothalamus was increased in mice suffering from dysbacteriosis, which suggested that the appetite of HSHF-diet mice increased and bodyweight soared, as seen previously ${ }^{23}$. Immunofluorescent staining of microglia and astrocytes using antibodies to IBA-1 (Fig. 2C, G, Fig. S3B-B) and GFAP (Fig. 3A, Fig. S3B-A) showed that the number of astrocytes was reduced significantly and microglial cells were activated in mice suffering from dysbacteriosis. Expression of the cholinergic neuron of AChE (Acetylcholinesterase) (Fig. 3B, $p<0.05$ ), Amphiphysin (AMP) (Fig. 3B, $p<0.05$ ), Acetylcholine receptor of CHRNB1 and CHRNA1 measurement showed that the cholinergic system was affected.
Expression of TNF- $\alpha(p<0.05)$ (Fig. 3C) and NF-kB p65 $(p<0.05)$ (Fig. 3C) was activated, whereas expression of PPAR- $\gamma$ and IL-2 showed no obvious differences $(p>0.05)$ (Fig. 3C). These data demonstrated that atrophy, inflammation, or the immune response were imbalanced in the brains of mice with HSHF diet-induced dysbacteriosis.

\section{The HSHF diet accelerates circRNAs degradation in mice brains}

A total of 7362 circRNAs were identified in the brains of mice fed a HSHF diet (Fig. 3). Among them, 287 differentially expressed circRNAs were in the control group vs. model group (fold change $>1.50, p<0.05$ ) (Fig. 3D, and Table 1, circBase): expression of 88 circRNAs was upregulated, and expression of 199 circRNAs was downregulated. This finding indicated that the circRNAs had different expression profiles in slender and obese mice. Bioinformatics analysis of differentially expressed circRNAs showed that dysbacteriosis could influence the brain in terms of metabolism, synaptic
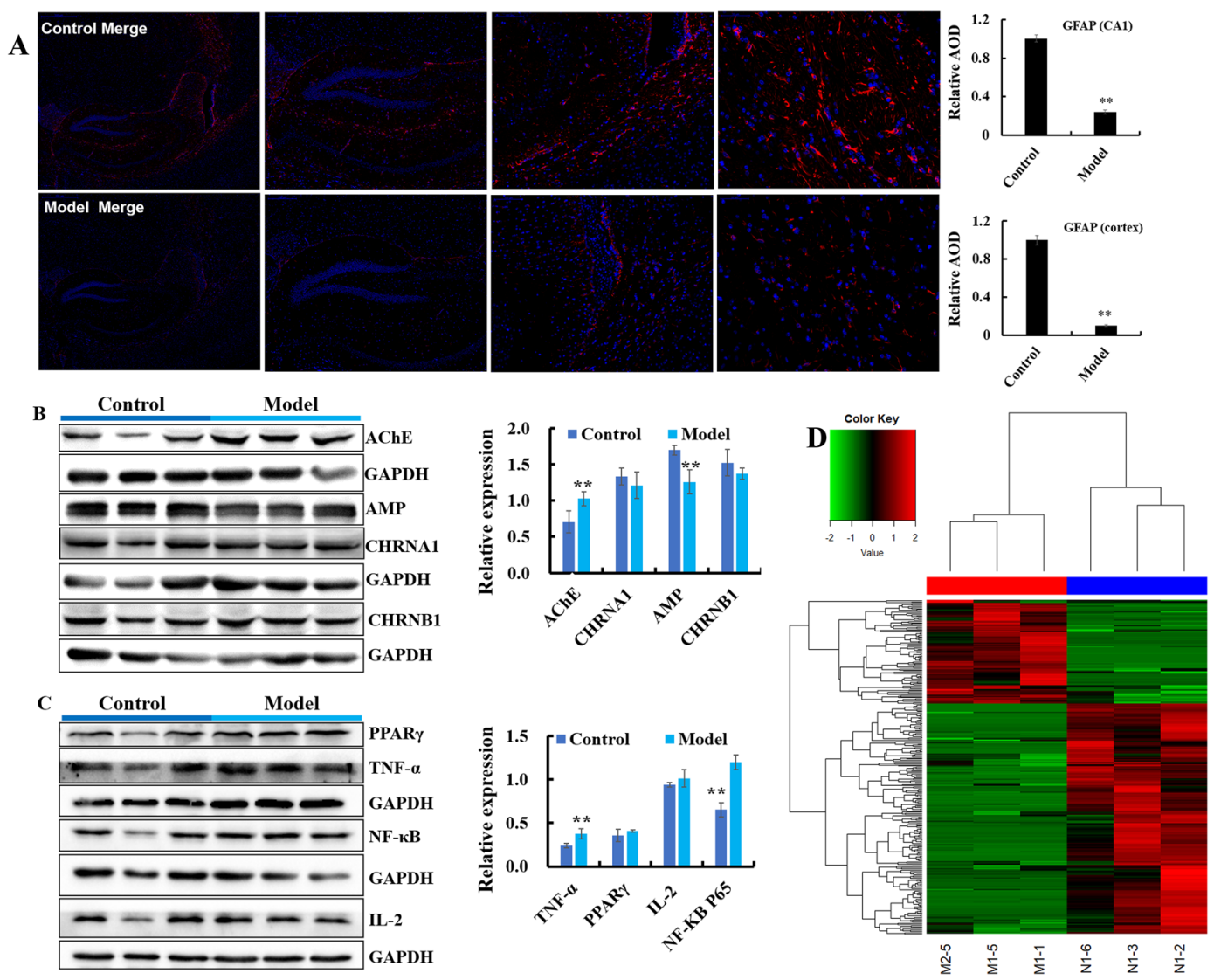

Fig. 3 Dysbacteriosis affects brain functions and circRNA sequencing in mice fed a diet high in sugar and fat for 4 months. A Samples were stained using an immunofluorescent antibody of GFAP, see also Fig. S3B-a. B, C Dysbacteriosis implicated the cholinergic system, inflammation and immune system in the brain. D Heatmap showing different expression of circRNAs in brain samples from mice fed a diet high in sugar, see Table 1. Data are the mean \pm SD of 3 independent experiments. ${ }^{*} p<0.05$ and ${ }^{* *} p<0.01$ vs. the model group by one-way ANOVA, followed by the Holm-Sidak test. 


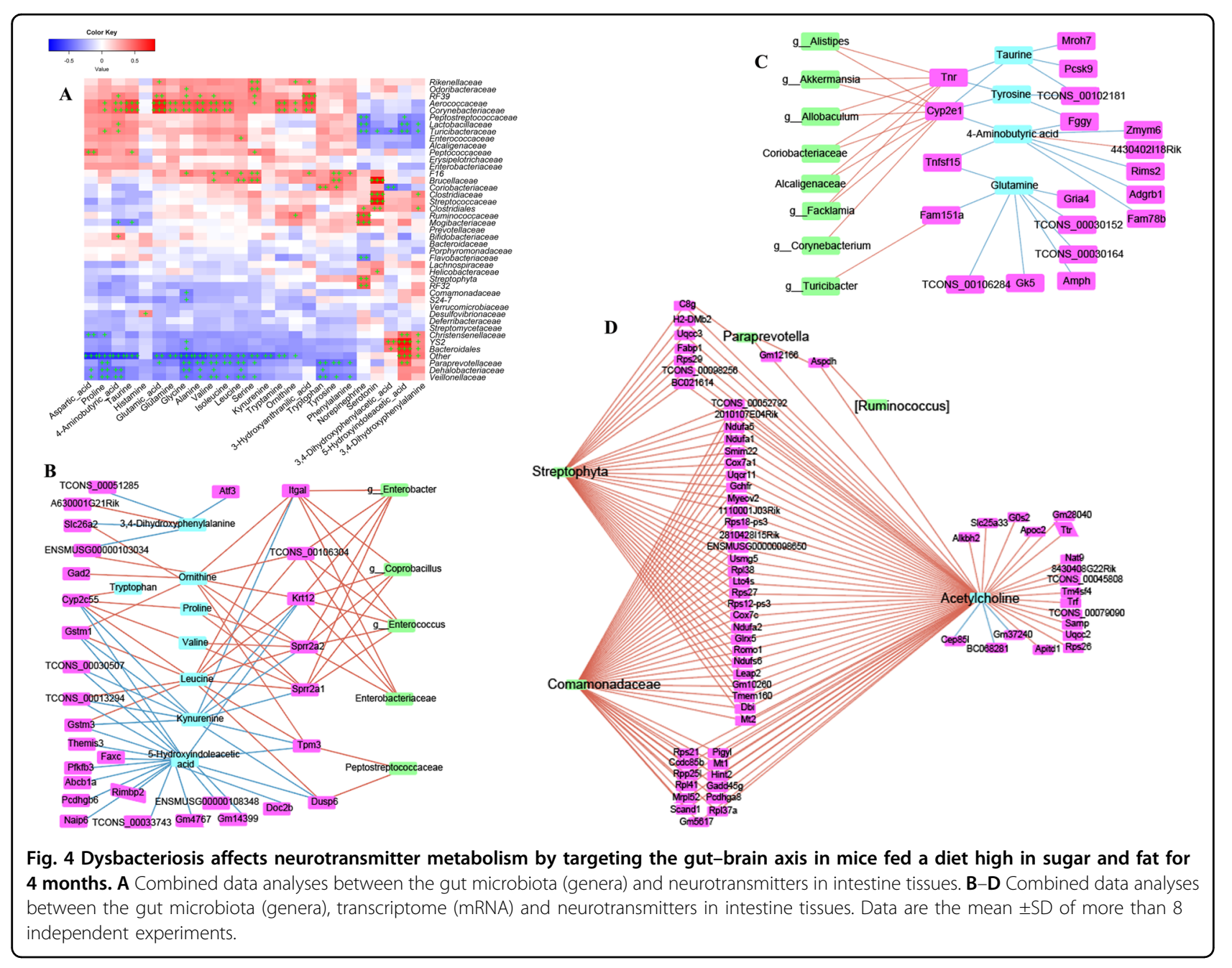

transmission and plasticity, and endogenous hormone levels. These actions would affect the activities and functions of the brain, but also affect heart function (e.g., circadian rhythm, dilated cardiomyopathy, and arrhythmogenic right ventricular cardiomyopathy). These data demonstrated that dysbacteriosis could affect brain circRNA-seq directly or indirectly.

\section{Networks interaction of the GM, neurotransmitters, intestinal mRNAs, and brain circRNAs} Network interaction of the GM and intestinal neurotransmitters

To identify the influences of the GM on neurotransmitters in the intestine, combined data analyses of the $\mathrm{GM}$ and neurotransmitters in fimo were done $\left(R^{2}>0.85\right)$. Some members of Aerococcaceae, Corynebacteriaceae, Brucellaceae, F16, Paraprevotellaceae, Veillonellaceae, and Dehalobacteriaceae were the key bacteria related to the production and/or secretion of neurotransmitters (Fig. 4A). They influenced proline, glycine, leucine, and serine (Fig. 4A). Phenylalanine, tryptophan, and tyrosine were influenced by bacterial in Veillonellaceae, Dehalobacteriaceae,
Paraprevotellaceae, Coriobacteriaceae, Brucellaceae, and F16, but how these bacteria produce them, and then participate or intervene in host metabolic pathways, merits further study.

The norepinephrine level was influenced by bacteria from Streptophyta, RF23, Flavobacteriaceae, Lactobacillaceae, Ruminococcaceae, Turicibacteraceae, Clostridiales, Mogibacteriaceae and Peptostreptococcaceae (Fig. 4A). The 5-hydroxy tryptamine (5-HT) level was influenced by bacteria from Streptococcaceae, Helicobacteraceae, Clostridiales, Turicibacteraceae, Clostridiaceae, and Brucellaceae (Fig. 4A). The level of 5Hydroxyindoleacetic acid (5-HIAA), the primary metabolite of $5-\mathrm{HT}$, was influenced by bacteria from YS2, Lactobacillaceae, Turicibacteraceae, Christensenellaceae, Peptostreptococcaceae, Paraprevotellaceae, and Bacteroidales (Fig. 4A).

Upon combination with data for intestinal mRNAs, we found that intestinal neurotransmitters also influenced intestinal genes, and then bacteria. As shown in Fig. 4B, leucine was positive to the mRNA of glutathione 
S-transferase Mu 3 (Gstm3), integrin subunit alpha L (Itgal), Krt12, small proline rich protein 2 A 2 (Sprr2a2), small proline rich protein 2 A 1 (Sprr2a1), tropomyosin 3 (Tpm3), dual specificity phosphatase 6 (Dusp6), and then to bacteria from Coprobacillus, Enterococcus, Enterobacter, Enterobacteriaceae, and Peptostreptococcaceae. The gene of cytochrome P450 family 2 subfamily E member 1 (Cyp2e1) influenced taurine, tyrosine, and 4Aminobutyric acid (Fig. 4C), and then became involved with the bacteria of Akkermansia, Allobaculum, Coriobacteriaceae, Alcaligenaceae, Corynebacterium Alistipes, and Facklamia (Fig. 4C). Tenascin R was involved with several types of bacteria (Fig. 4C).

Network interaction of the GM and brain neurotransmitters

The bacteria of Flavobacteriaceae, Porphyromonadaceae, Bifidobacteriaceae, Bacteroidaceae, Desulfovibrionaceae Enterococcaceae, Prevotellaceae, Lactobacillaceae and Peptostreptococcaceae could interact with most brain neurotransmitters: isoleucine, valine, histamine, serine, proline, leucine, phenylalanine, tryptophan, tyrosine, glutamic acid, 3,4-Dihydroxyphenylalanine, kynurenine, 5Hydroxyindoleacetic acid, tryptamine, glycine, and ornithine (Fig. 5A). The brain neurotransmitters homovanillic acid and 3,4-Dihydroxyphenylacetic acid were influenced by bacteria from Bacteroidales, YS2, Paraprevotellaceae, Enterobacteriaceae and Bacteroidaceae (Fig. 5A).

The "cholinergic hypothesis" has an important role in $\mathrm{AD}$ therapy $\mathrm{y}^{24}$. The degenerative dysfunction of cholinergic neurons is a partial cause of memory deficit in dementia patients. The neurotransmitter acetylcholine (ACh) in the brain was implicated with several intestinal genes (Fig. 4D, Table S1 and enrichment analysis using the KEGG database), and might be intervened and/or promoted by bacteria from Paraprevotella, [Ruminococcus], Streptophyta and Comamonadaceae (Fig. 4D). These data indicated that the GM has complex interactions with the host cholinergic system. We discovered that the norepinephrine level in the brain could: (i) influence bacteria of Peptostreptococcaceae, Sutterella, Flavobacteriaceae and Coprobacillus; interact with bacteria of Enterococcus, Enterobacteriaceae, and Enterobacter, and then interact with 3,4-dihydroxyphenylacetic acid; interact with bacteria from Lactobacillus, and then influence 5hydroxyindoleacetic acid (Fig. 5B).

Glutamine, isoleucine, phenylalanine, tyrosine, leucine, tryptophan, proline and valine did not interact with a single type of bacteria (Fig. 5B). Hence, the interactions between GM, neurotransmitters, and host gene are complicated. More other complex interactions between the GM, mRNA-seq of intestinal tissue, and neurotransmitters of brain tissues are shown in Figs. 4 and 5. The neurotransmitters ACh, 4-aminobutyric acid, 5-hydroxyindoleacetic acid (5-HIAA), glutamine, serotonin, leucine, and kynurenine may also have important roles in the brain-GM axis, or the GM may be indispensable for the synthesis and secretion of neurotransmitters: the exact mechanism needs much more work.

\section{Network interaction of the GM, brain neurotransmitters, and circRNAs}

Next, the interactions of the GM, neurotransmitters, and circRNAs in brain tissues were analyzed. The ACh level in brain tissues was important for the interactions between the brain circRNA of chr5_21278044/21281602_+, chrX_162934286/162941844_+ and chr16_37121798/ 37129964_-, and bacteria from Paraprevotella, Helicobacteraceae, Erysipelotrichaceae, [Ruminococcus] and Streptophyta (Fig. 5C). The neurotransmitters 5-HIAA, leucine, 3,4-dihydroxyphenylacetic acid, kynurenine, ornithine, tryptamine, $\mathrm{ACh}$, and tyrosine were the main metabolites influencing the GM and circRNAs (Fig. 5D), or they could influence multiple microorganisms and circRNAs (Fig. 5). Much more experimental evidence is needed to explain these associations.

5-HIAA is the main metabolite of 5-HT. A recent study showed that 5 -HT directly stimulates and inhibits the growth of commensal bacteria in vitro, and exhibits concentration-dependent and species-specific effects ${ }^{25}$. Some bacteria may have important roles in the conversion of 5-HT to 5-HIAA. Bacteria from the family Peptostreptococcaceae could affect the mRNA of Tpm3 and Dusp6 (Figs. 4 and 5). Research has revealed a genetic proof-of-function for choline acetyltransferase (ChAT) in $\mathrm{T}$ cells during viral infection and identified a pathway of $\mathrm{T}$-cell migration that sustains antiviral immunity $^{26}$. Levels of ACh and ChAT were influenced primarily by bacteria from Helicobacteraceae, Paraprevotella, [Ruminococcus], Erysipelotrichaceae, Comamonadaceae, and Streptophyta (Figs. 4 and 5). The role of the GM in TMAO byproducts from choline has been demonstrated $^{27}$, and TMAO has been shown to be harmful in several diseases ${ }^{28}$. Therefore, the GM acts as a bridge to contribute to host nutrition/health by regulating the metabolism. The above-mentioned data, together with data from other studies, suggest that gut dysbacteriosis could impact circRNA sequences in the brain.

\section{TMAO byproducts influence circRNA levels in BV2 cells}

In this additional experiment, expression of some circRNAs in a murine microglial cell line (BV2) was monitored after incubation with TMAO $(18.45 \mathrm{mg} / \mathrm{mL}) \quad(p<0.05)$ (Fig. 6A). Expression of MAOA (Amine oxidase [flavincontaining] A), MAOB, COMT (Catechol O-methyltransferase), AChE, AMP, CHRNA1 and CHRNB1 was influenced by this concentration of TMAO $(p<0.05)$ (Fig. 6B), but a low concentration of TMAO did not cause 


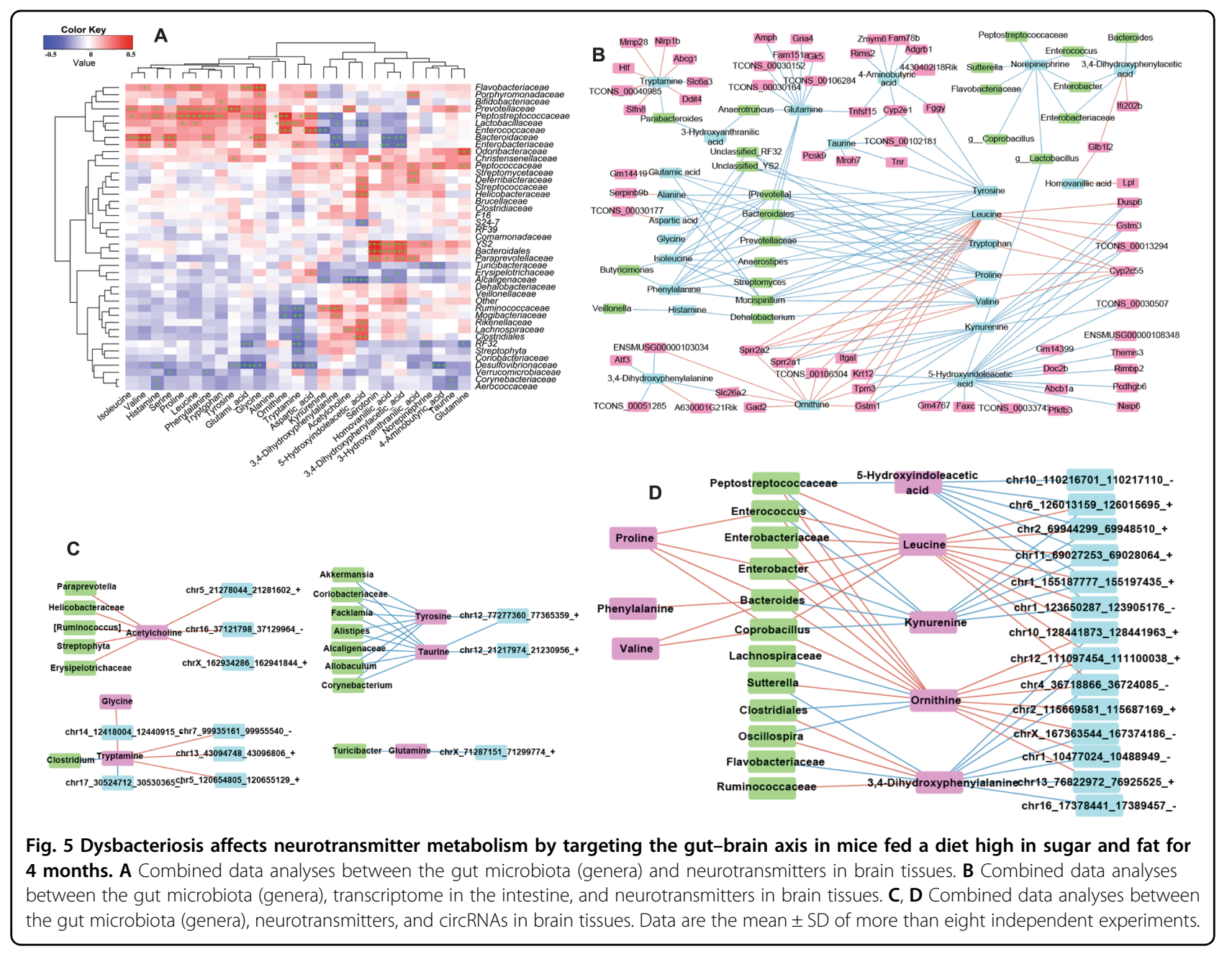

obvious damage to BV2 cells. Percentage inhibition in BV2 cells did not differ at a TMAO concentration $<16 \mathrm{mg} / \mathrm{mL}$ (Fig. 6A). Expression of some circRNAs, including circNF1419 and circ_0001239, was influenced by a low concentration of TMAO $(p<0.05)$ (Fig. 6C), indicating that some circRNAs might be sensitive responsive signaling molecules to TMAO. These data suggested that microbial metabolites might influence the formation and degradation of host circRNAs.

Levels of MAOA, MAOB, and COMT were affected after TMAO administration to BV2 cells (Fig. 6). Levels of some monoamine neurotransmitter were changed in mice fed the HSHF diet (Figs. 4 and 5). Taken together, these data suggested that behavior and stress would be influenced and, in these processes, the GM had an accelerant role. Therefore, the GM decides the transformation efficiency from choline to TMAO. The latter influences the levels of MAOA, MAOB, and COMT, which participate in the regulation of metabolism of neuroactive and vasoactive amines in the central nervous system (CNS) and peripheral tissues.
circRNAs spliced from Rims1/Syt1/Unc13b could be enriched in the ACh release cycle (Fig. 7A). Levels of some of these circRNAs were measured in the brain tissues from different mice after choline treatment. Choline and/or its metabolite could change circRNA expression (Fig. 7B), which suggested that the GM also influences the absorption and conversion of choline. Pecoraro and colleagues suggested that a high-affinity PAC1 (Proteasome assembly chaperone 1) receptor presynaptically modulates hippocampal glutamatergic transmission acting through AChE in hippocampal $\mathrm{CA}^{29}$, indicating that choline plays an important part in neurotransmitter homeostasis.

\section{The GM plays an important part in the conversion rate of choline to TMAO}

The GM plays an important part in lipid homeostasis in blood, and in the causes and development of nervoussystem diseases. In this study, the levels of total cholesterol (T-CHO, Fig. 7Ca), triglyceride (TG, Fig. 7Cb) and lowdensity lipoprotein (LDL, Fig. 7Cc) were also increased 

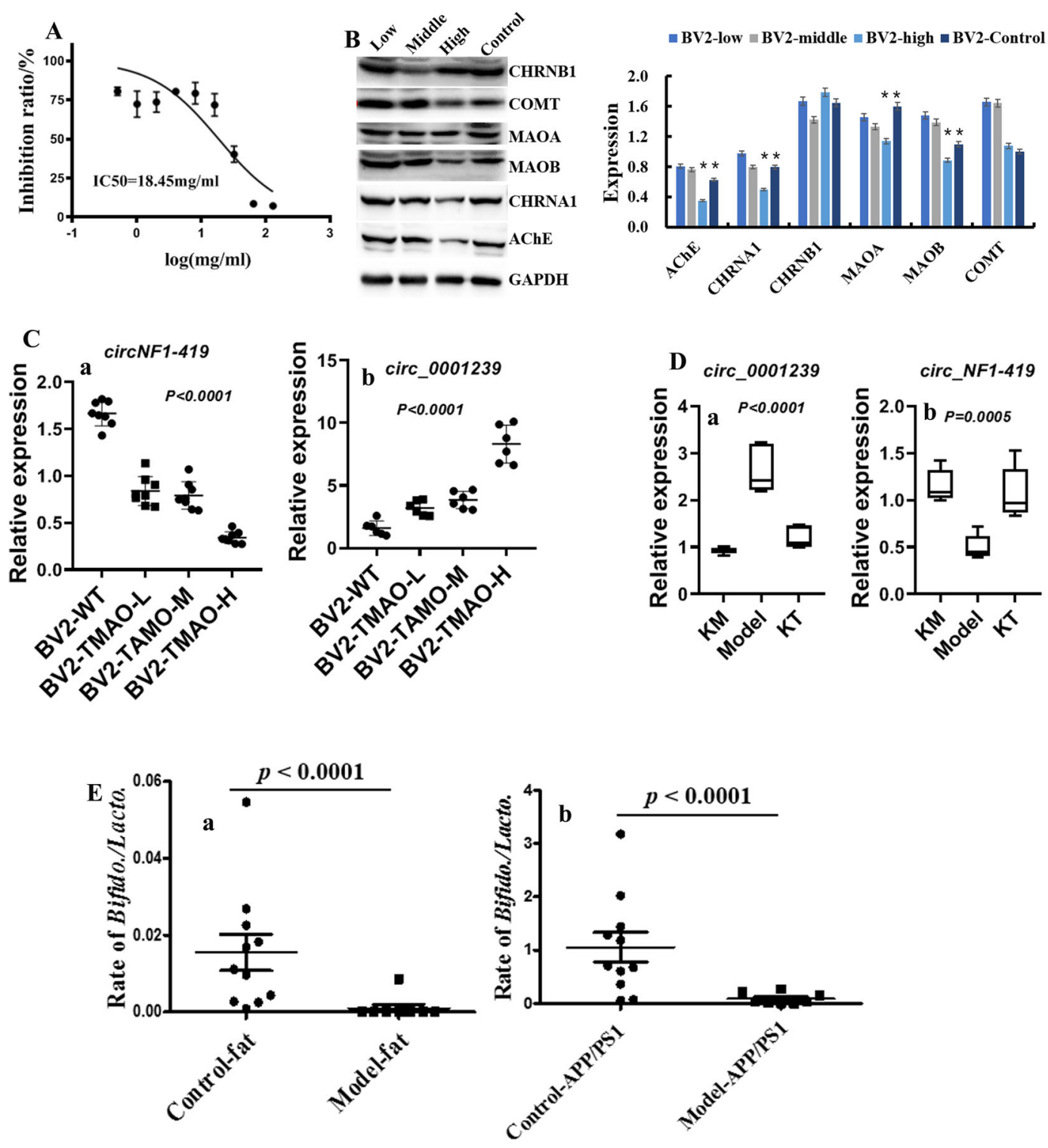

Fig. 6 Effects of TMAO on BV2 cells. A Effects of TMAO on proliferation of BV2 cells, measured using the MTT assay. B Expression of MAOA, MAOB, COMT, AChE, AMP, CHRNA1, and CHRNB1 in TMAO-damaged BV2 cells. C Expression of circNF1-419 and circ_0001239 in TMAO- damaged BV2 cells. D Expression of circNF1-419 and circ_0001239 in Kombucha tea-treated mice. E Ratio of abundance of Bifidobacterium species and Lactobacillus species was imbalanced in mice fed a HSHF diet and in AD-like mice. Data are the mean \pm SD of more than five independent experiments (and three independent experiments when using western blotting). ${ }^{*} p<0.05$ and ${ }^{* *} p<0.01$ vs. the model group by one-way ANOVA, followed by the Holm-Sidak test.

$(p<0.05)$, and the levels of serum TMAO (Fig. 3C), when compared to the HSHF-diet group $(p<0.05)$, and the highdensity lipoprotein (HDL, Fig. 7Cd) were decreased $(p<0.05)$.

Rats treated with TMA could put on weight continuously (Fig. 8Aa), had increased levels of TMAO in serum (Fig. 8Ab) and increased levels of total $\mathrm{T}-\mathrm{CHO}$ (Fig. 8Ac), TG (Fig. 8Ac) and LDL (Fig. 8Ac) $(p<0.05)$ when compared with those in the model group $(p<0.05)$, whereas the HDL content was reduced $(p<0.05)$ (Fig. 8Ac). Data for sequencing of the $16 \mathrm{~S}$ rRNA gene showed that TMA decreased the OTUs of bacteria from Verrucomicrobia, TM7 and Acidobacteria $(p<0.05)$ (Fig. 8B), while increasing the OTUs for bacteria from Tenericutes, Deferribacteres, Actinobacteria, and cyanobacteria $(p<0.05)$ (Fig. 8B). At the genus level, the OTUs of bacteria from Bifidobacterium, Aerococcus, Facklamia, Blautia and Enterobacteriace were increased by TMA 

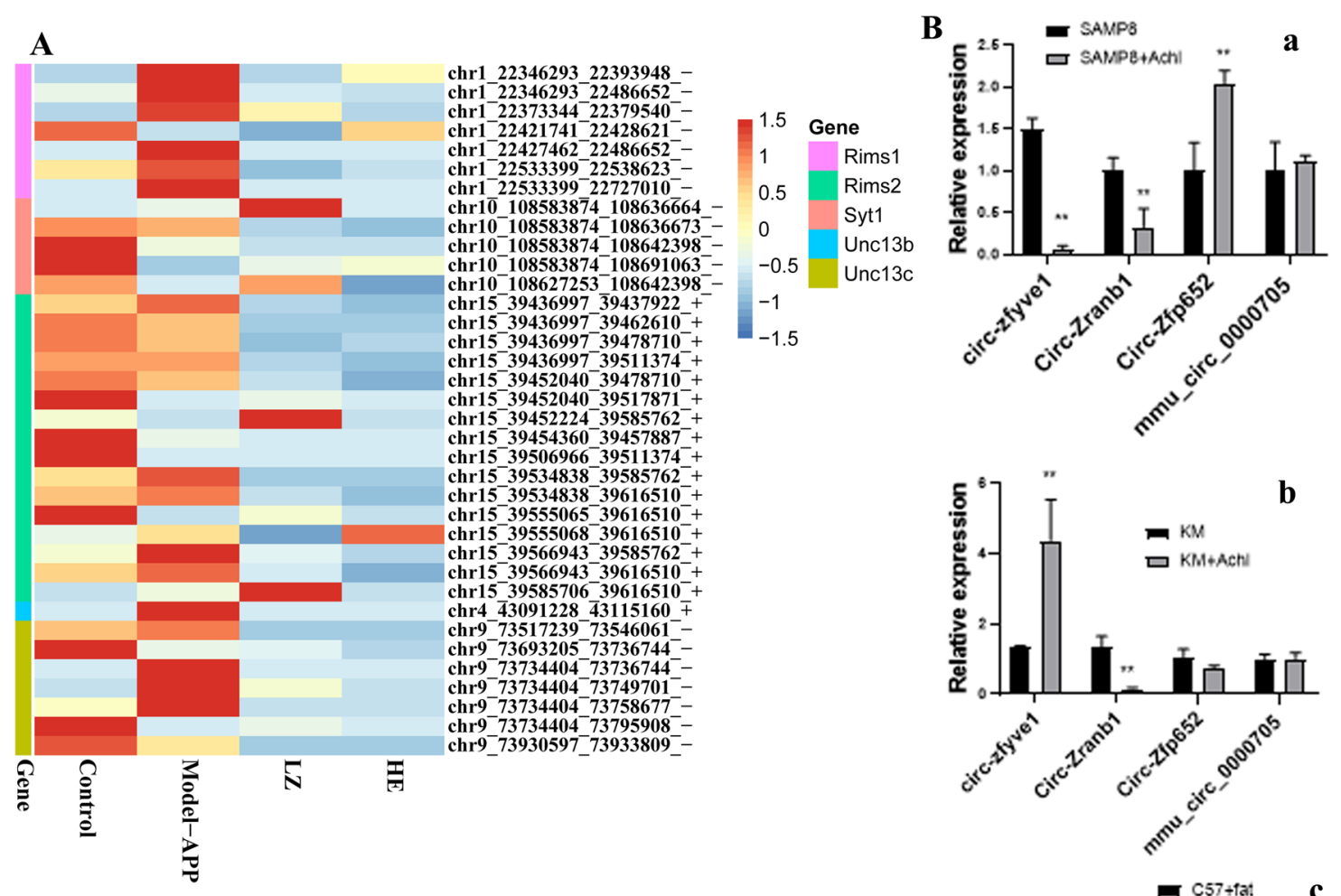

chr15-39454360-39457887-

chr15_39506966 39511374 -

chr15_39534838_39585762 +

chr15_39534838_39616510-

chr15_39555065_39616510 +

chr15_39555068_39616510 +

chr15_39566943_39585762 +

chr15_39566943_39616510 -

chr15 39585706_39616510 +

chr4_ $\mathbf{4 3 0 9 1 2 2 8} \overline{43115160}{ }^{+}$

chr9 73517239 - 73546061 -

chr9-73693205_73736744--

chr9-73734404-73736744--

chr9-73734404-73749701--

chr $9^{-73734404} 73758677_{-}^{-}$

chr9_73930597_73933809--
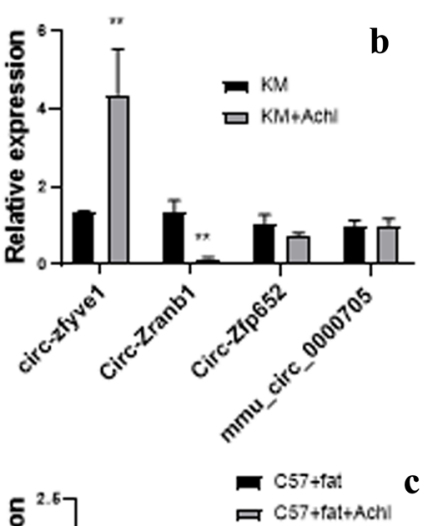

C
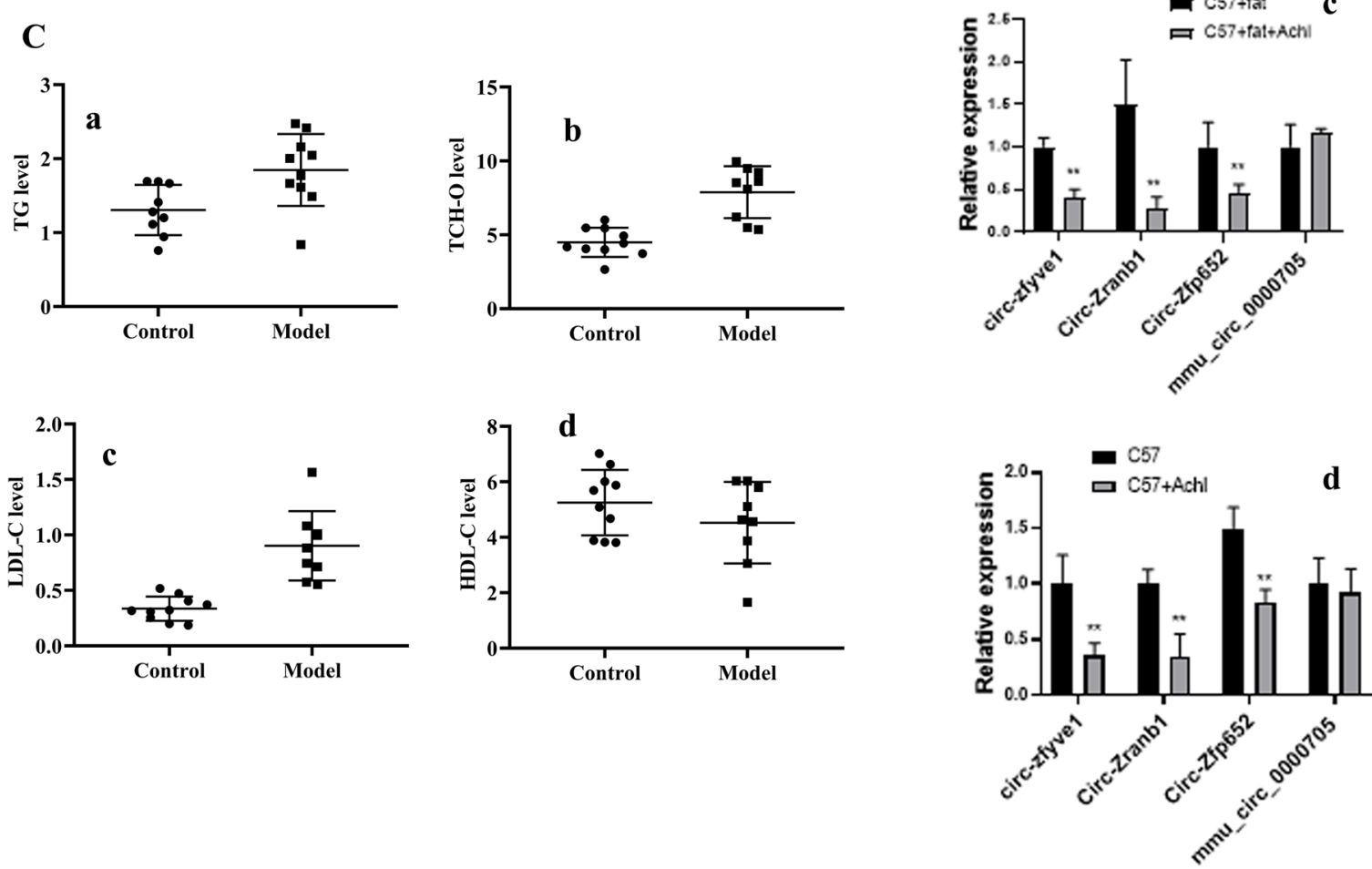

Fig. 7 Effects of choline on circRNA expression and lipid metabolism. A Relative expression of circRNAs spliced from Rims $1 / 5 y t 1 /$ Unc13b enriched in the acetylcholine release cycle. B Expression of circRNAs in choline-treated mice using RT-qPCR. C Levels of total cholesterol (T-CHO), triglyceride $(\mathrm{TG})$, low-density lipoprotein $(\mathrm{LDL})$, and high-density lipoprotein $(\mathrm{HDL})$ in mice fed a diet high in sugar and fat. Data are the mean $\pm \mathrm{SD}$ of more than 6 independent experiments. ${ }^{*} p<0.05$ and ${ }^{* *} p<0.01$ vs. the model group by one-way ANOVA, followed by the Holm-Sidak test. 

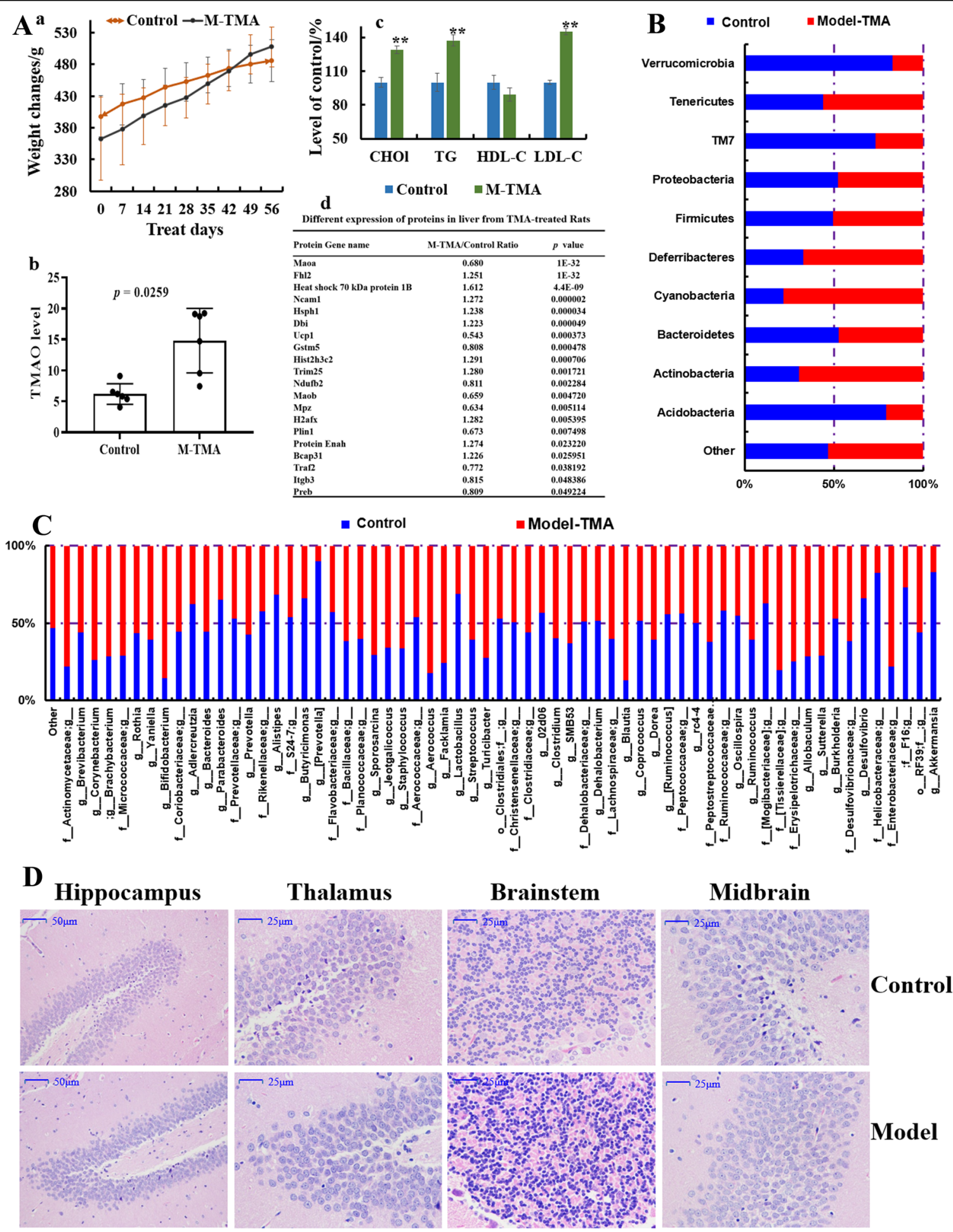

Fig. 8 Effects of trimethylamine (TMA) on rats. A Effects on bodyweight changes (a), levels of T-CHO, LDL, HDL, and TG (b), levels of TMAO (c), and protein expression in liver tissues after intragastric administration of TMA at $0.2 \mathrm{~mL} / 100 \mathrm{~g} / \mathrm{d}$ of $2.5 \%$ for 6 weeks. B Effects on the gut microbiota at the phylum level, and $(\mathbf{C})$ at genus level. D Pathologic changes in hippocampus, thalamus, brainstem, and midbrain after TMA administration. Data are the mean or mean \pm SD of more than 8 independent experiments. ${ }^{*} p<0.05$ and ${ }^{* *} p<0.01$ vs. the model group by one-way ANOVA, followed by the Holm-Sidak test. 
administration $(p<0.05)$ (Fig. 8C), whereas those of bacteria from Prevotella, Lactobacillus, Helicobacteraceae and Akkermansia were decreased significantly $(p<0.05)$ (Fig. 8C), which indicated that excessive TMA in the diet can induce dysbacteriosis. Histopathologic changes in the hippocampus, thalamus, brainstem, and midbrain of the TMA-induced group compared with those in the control group (Fig. 8D) included cell shrinkage, cytoplasmic vacuolar changes, blurred and indistinct cell boundaries, and scattered damaged tissue blocks. Also, the differential expression of proteins in the liver from TMA-treated rats revealed that TMA induced damage in the host. For example, the level of mitochondrial brown fat uncoupling protein 1 was decreased after treatment with excess TMA, together with a decrease in Ndufb2 expression (Fig. 8Ad), which indicated that energy metabolism was imbalanced due to dysfunction of NADH dehydrogenase activity and oxidoreductase. Meanwhile, Ncam1 expression was upregulated (Fig. 8Ad), suggesting that neurite outgrowth, synaptic plasticity, as well as learning and memory might have been influenced. Increased expression of heat shock $70 \mathrm{kDa}$ protein $1 \mathrm{~B}$ and $\mathrm{B}$-cell receptor-associated protein 31 (Fig. 8Ad) indicated that the host stress response was activated and immune-system function might have been compromised. In agreement with results from other studies, TMA resulted in negative consequences for the host $^{29,30}$ and, in the present study, TMA led to negative consequences for the nervous system.

\section{C. albicans and $K$. pneumoniae influence the cholinergic system in mice}

We wished to ascertain the potential routes of communication/interaction between the host and its resident bacteria with regard to neurotransmitter metabolism and brain function. $C$. albicans and $K$. pneumoniae were administered (i.g.) to normal C57 mice as monotherapy or in combination. Expression of AChE, AMP, CHRNA1, CHRNB1, and GAD65 was changed in the intestine $(p<$ 0.05) (Fig. 9A) and brain (Fig. 9B), especially in the $C$. albicans-treated group in the intestine. These data indicated that changes in two core microorganisms influenced GM-mediated compounds and neurotransmitters.

\section{Discussion}

Increasing numbers of studies have indicated that the GM is closely related to disorders of the nervous system, including autism ${ }^{31}$, depression ${ }^{32}$, schizophrenia ${ }^{33}$, anorexia nervosa ${ }^{34}$, multiple sclerosis ${ }^{35}$, epilepsy ${ }^{36}, \mathrm{PD}^{37}$, and $\mathrm{AD}^{18}$. In recent years, evidence has shown that circRNAs may be important for brain function: CDR1 is found at a high level in mammalian neurons ${ }^{38}$, and the circRNA transcripts from neuron genes can accumulate during aging in Drosophila ${ }^{39}$. Hentze and colleagues showed that circRNAs are dynamic during the activities of daily life ${ }^{40}$.
Also, circRNAs are expected to be biomarkers or drug targets for neurodegenerative diseases, and studies have shown that the CDR1 level is reduced in $\mathrm{AD}^{38}$. Here, we demonstrated that gut dysbacteriosis can implicate the brain circRNA-sequencing directly or indirectly.

Previously, using in an overexpressed circNF1-419 adeno-associated virus system, we showed that circRNAs in the brain influenced the cholinergic system of the brain, and changed the GM composition, intestinal homeostasis/physiology, and even the GM trajectory in newborn mice ${ }^{41}$. Another system, circZCCHC11 (mmu_circ_0001239), showed a "sponge" miRNA function and mediated a series of chain reactions in the brain, which then influenced gut function and GM engraftment from their parent ${ }^{41}$. Those data demonstrated a link between circRNAs and the GM, enlarged the microbiome-transcriptome linkage library, and provided additional information on the gut-brain axis. Those data and the observations mentioned above indicated that the GM influences the conversion rate of choline to TMAO, and that the TMAO level influences the cholinergic system. In an additional experiment, expression of some circRNAs in the BV2 cell line was measured after incubation with TMAO, which indicated that some circRNAs might be sensitive responsive signaling molecules to TMAO. We concluded that microbial metabolites influenced the formation and degradation of host circRNAs.

Studies have demonstrated that catalyzes the oxidative deamination of biogenic and xenobiotic amines, and has important functions in the metabolism of neuroactive and vasoactive amines in the CNS and peripheral tissues. For example, MAOA preferentially oxidizes biogenic amines such as $5-\mathrm{HT}$, norepinephrine, and epinephrine. MAOB preferentially degrades benzylamine and phenylethylamine. COMT catalyzes the transfer of a methyl group from S-adenosylmethionine to catechol substrates such as the neurotransmitters dopamine, epinephrine, and norepinephrine, and degrades catecholamine transmitters ${ }^{42}$. All of the above-mentioned monoamine neurotransmitters have indispensable roles in the regulation of CNS functions.

We found that the levels of MAOA, MAOB, and COMT in BV2 cells were affected after TMAO administration, and that the levels of some monoamine neurotransmitters were changed in mice fed the HSHF diet. These data suggested that behavior and stress would be influenced and that, in these processes, the GM had an accelerant role. Therefore, we concluded that the GM decided the transformation efficiency from choline to TMAO. The latter then influenced the levels of MAOA, $\mathrm{MAOB}$, and COMT, which participate in the regulation of metabolism of neuroactive and vasoactive amines in the CNS and peripheral tissues. How circRNAs are involved in this process merits further investigation. 

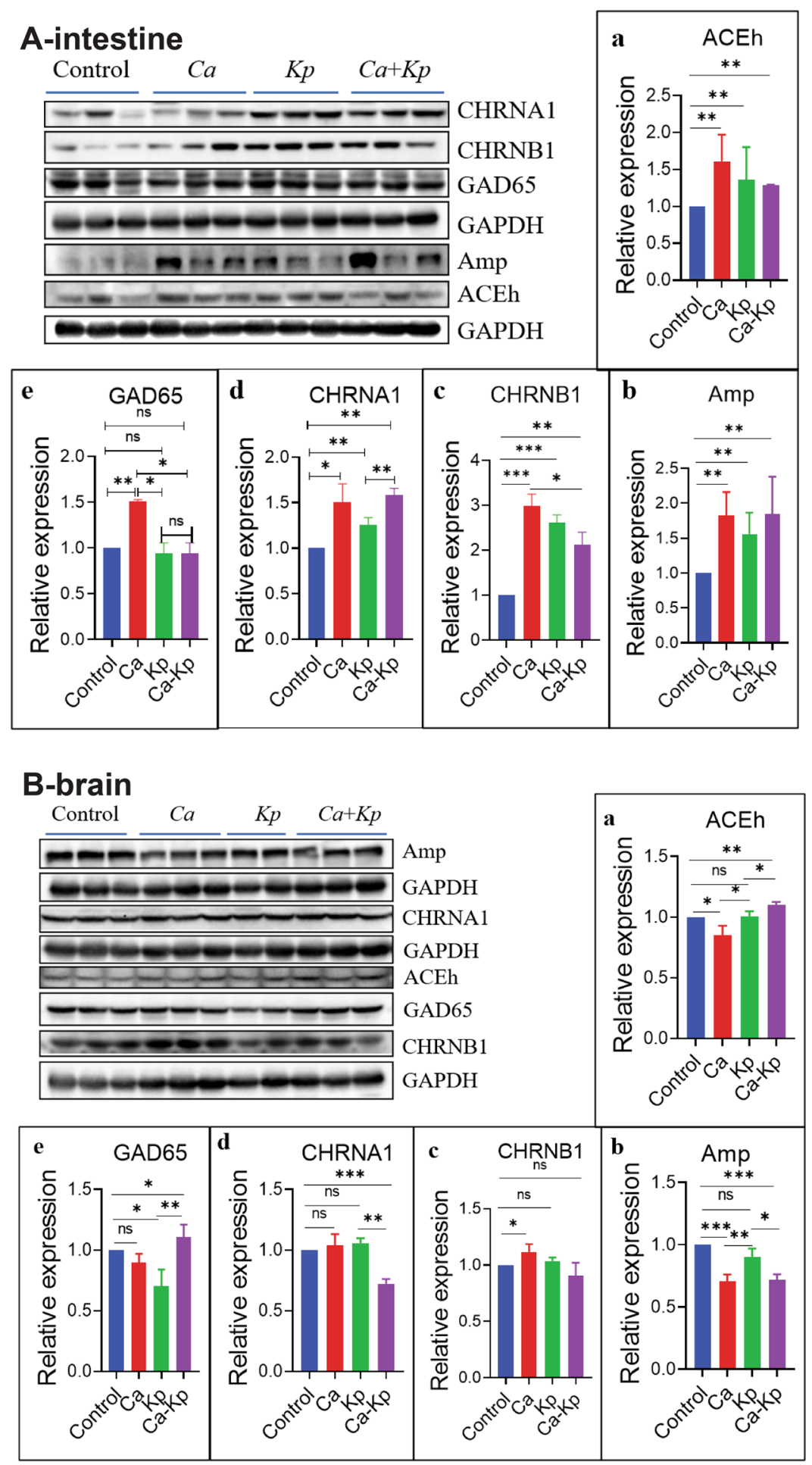

Fig. 9 C. albicans and Klebsiella pneumoniae influence the cholinergic system in mice. A Expression of AChE, AMP, CHRNA1, CHRNB1, and GAD65 were changed in the intestine $(\mathbf{A}, p<0.05)$ and brain $(\mathbf{B})$. Data are the mean \pm SD of three independent experiments. ${ }^{*} p<0.05$ and ${ }^{* *} p<0.01$ vs. the model group by one-way ANOVA, followed by the Holm-Sidak test. 
A major goal of any microbiome study is to move beyond correlation, and parse out potential routes of communication/interaction between the host and its resident bacteria. To identify their communication/ interaction, the species level was identified using metagenomics sequencing. Twelve species of Archaea were detected, including Methanobrevibacter sp. AbM4 (781.63-fold changes compared with control, Fig. S4A), Methanosarcina sp. MTP4 (1.21-fold, Fig. S4A), and especially Methanosalsum zhilinae, Methanomethylovorans hollandica, Candidatus Methanomethylophilus alvus, and Thermococcus cleftensis, which were new residents (Fig. S4A). Most are methanogens (anaerobic prokaryotes from the domain Archaea that utilize hydrogen to reduce carbon dioxide, acetate, and various methyl compounds to methane ${ }^{43}$ ). Methanogens described in the human microbiota include Euryarchaeota (including M. smithii, M. oralis, $M$. arbophilus, $M$. massiliensis, $M$. luminyensis, M. stadtmanae, Ca. M. alvus, and Ca. M. intestinalis ${ }^{44}$ ). Methanogens are emerging pathogens associated with abscesses in the brain and muscles ${ }^{44}$. They have been implicated in dysbiosis of the oral microbiota, periodontitis, and peri-implantitis ${ }^{43}$. They have also been associated with dysbiosis of the digestive-tract microbiota linked to metabolic disorders (anorexia, malnutrition, and obesity) and with lesions of the digestive tract (colon cancer) ${ }^{43}$. One clinical investigation showed that a negative association between methane concentrations in breath and anthropometric biomarkers of obesity ${ }^{45}$, and that methane significantly decreased the neurological deficit induced by cerebral ischemia and reperfusion via the antioxidant pathway of PI3K/Akt/HO- $1^{46}$. Special diets have been used to change anaerobic prokaryotes to involve the digestive and nervous systems ${ }^{47}$. ACh is a neurotransmitter in mammalian central and peripheral cholinergic nervous systems. However, it is also widely expressed in non-neuronal animal tissues as well as in plants, fungi, and bacteria, where it is likely involved in the transport of water, electrolytes and nutrients ${ }^{48}$. With the changes in levels of methanogens, based on histopathology, we found that the colon and brain was damaged; neurotransmitter levels were also changed.

Mycobiota are crucial for human health ${ }^{49}$. Surprisingly, a small number of species can trigger huge changes in the human body ${ }^{50}$. Dysbiosis of and invasion by mycobiota can cause disease in different parts of the body ${ }^{51,52}$. Meanwhile, the body also produces corresponding immune changes upon mycobiota infection ${ }^{53}$. Several recent studies have made a connection between intestinal mycobiota and the human immune system ${ }^{52,53}$. In HSHFdiet mice, 10 species of Eukaryota were detected. For example, the relative abundance of Kluyveromyces lactis, Leishmania major, Saccharomycetales species and Theileria orientalis was different $(p<0.05)$ (Fig. S4B).
C. albicans is a very common yeast found in the gastrointestinal tract and oral cavity, and can occasionally cause oral-cavity ulcers. C. albicans can pass readily through the blood-brain barrier, where it can cause asymptomatic infection in the cortex, form fungal- induced glial granulomas, and cause short-term memory disorders ${ }^{54}$. Candida dubliniensis is an opportunistic fungal pathogen. Bacher and colleagues revealed that human immunity based on T-helper 17 cells against fungi is reliant on cross-reactivity against C. albicans ${ }^{53}$. Witchley and coworkers showed that programs of $C$. albicans morphogenesis control the balance between gut commensalism and invasive infection ${ }^{55}$. Neuronal $\mathrm{ACh}$ and non-neuronal $\mathrm{ACh}$ have been demonstrated to modulate the inflammatory response: $\mathrm{ACh}$ protects against $C$. albicans infection by inhibiting biofilm formation and promoting hemocyte function in a model of Galleria mellonella infection $^{56}$. Hence, C. albicans and C. dubliniensis seem to have important roles in host immunity. In comparison with normal groups, the relative abundance of $C$. dubliniensis was increased (Fig. S4B), and levels of proinflammatory mediators were upregulated, upon consumption of the HSHF diet (Fig. 3). Also, in C. albicans- and K. pneumoniae-treated C57 mice, expression of AChE, AMP, CHRNA1, CHRNB1, and GAD65 were changed in the intestine (Fig. 9A) and brain (Fig. 9A), especially in the C. albicans-treated group in the intestine. These data indicated that changes in a few microorganisms could influence microbiota-mediated compounds (including neurotransmitters).

Cooperation within the GM is complex and important for human health, such as mood and bodyweight control. In most cases, the intestinal flora cooperate and influence each other. Cooperative phenotypes are thought to be at the core of microbial-community functions, including through quorum sensing, biofilm formation, and antibiotic resistance ${ }^{57,58}$. We found that with an increase in some Saccharomyces species who converted sugars to carbon dioxide and ethanol, the abundance of some methanogens was increased. The reason may have been because the metabolism of alcohol imbalances the proportion of NADH to NAD, galactose tolerance, TG synthesis, and lipid peroxidation (Figs. 3 and 7). Xu and colleagues revealed that chronic exposure to alcoholinduced GM dysbiosis, and was correlated with neuropsychic behaviors ${ }^{59}$. Furthermore, a metabolite from Saccharomyces, acetic acid, is the growth substrate of methanogens. The cooperation of these two microorganisms promotes food digestion/absorption, energy storage, and the accumulation of some harmful products $^{60}$. Studies have demonstrated that the PI3K-I/ Akt-mTOR signaling network can regulate anabolic processes such as the synthesis of lipids, fatty acids, and nucleotides, and requires an abundant supply of reducing 
power in the form of NADPH, and the growth factorstimulated PI3K-Akt-mTORC1 signaling network ${ }^{61,62}$, and the NADPH oxidase nox can be derived by gut microbiome ${ }^{63}$.

Twenty-two species of viruses were detected (Fig. S4C). The numbers of most of them were increased in the HSHF-diet group: Aureococcus anophagefferens virus, Glypta fumiferanae ichnovirus, Chrysochromulina ericina virus, mouse mammary tumor virus, Moloney murine sarcoma virus, murine leukemia-related retroviruses, murine leukemia virus, Mus musculus mobilized endogenous polytropic provirus, RD114 retrovirus, Shamonda orthobunyavirus, Tomato spotted wilt orthotospovirus, Cyprinid herpesvirus 1, Cyprinid herpesvirus 3, Abalone herpesvirus Victoria/AUS/2009, Cadicivirus A and Lactobacillus prophage Lj771. Analyses of circRNA sequences showed that the number of circRNAs with downregulated expression was much greater than the number of circRNAs with upregulated expression in HSHF diet-group mice. An identical trend was reported by Liu and colleagues. They found that circRNAs could be degraded by RNase $\mathrm{L}$ if induced by polyinosinic: polycytidylic acid or infected by a virus ${ }^{64}$. Furthermore, expression of retinoic acid-inducible gene I supported the notion that too much of a HSHF diet affects immunity and increases the risk of virus invasion $(p<$ 0.05) (Fig. S4D). Studies have revealed that Herpesvirus species are associated with $\mathrm{AD}^{65}$. Therefore, gut dysbacteriosis appears to be a critical factor in inducing changes in the circRNA-expression profile in the brain. Our results also suggest that the stability of circRNAs in terms of structure and quantity is needed for health (i.e., circRNAs may have non-negligible roles in physiological function/regulation in the organism). Also, studies have revealed that the bacteria, diet, and host genes are related to the invasion, infection degree, and drug resistance of viruses ${ }^{66,67}$.

A total of 622 species of bacteria were detected. The relative abundance of species was changed obviously in the model group (Fig. S5). Changes were observed in the abundance of Bifidobacterium, Lactobacillus, Bacteroides, Prevotella, Streptococcus and Clostridium species (Figure S5A-D). With an increase of Lactobacillus prophage Lj771 abundance (Fig. S4C), the abundance of most of the species of Lactobacillus was reduced significantly, including that of $L$. acidipiscis, L. agilis, $L$. allii, L. amylophilus, L. amylovorus, L. brevis, L. coryniformis, L. fermentum, L. gasseri, L. ginsenosidimutans, L. helveticus, L. jensenii, L. johnsonii, L. kefiranofaciens, L. paraplantarum, L. pentosus, L. plantarum, L. reuteri, L. rhamnosus, L. Ruminis and L. salivarius, which were found only in the control group (Fig. S4A-D). Simultaneously, the abundance of Bifidobacterium species, including that of B. adolescentis, B. asteroids, B. bifidum,
B. breve, and B. pseudocatenulatum, was also inhibited. In contrast, the abundance of most Bacteroides species was increased, including $B$. caccae, $B$. caecimuris, $B$. cellulosilyticus, B. dorei, B. fragilis, B. helcogenes, $B$. ovatus, B. salanitronis, B. Thetaiotaomicron and B. vulgatus. Phages may have been the reason why the ratio of Bifidobacterium species and Lactobacillus species was imbalanced in mice fed the HSHF diet (Fig. 6E). The abundance of Bifidobacteriaceae species and Lactobacillaceae species is crucial for homeostatic balance in the intestine. The relative abundance of Lactobacillaceae species was increased and that of Bifidobacteriaceae species was decreased in APP/PS1 mice (Fig. 6E). These data provide evidence that probiotics are beneficial for health under certain physiological conditions.

The abundance of members of the family Lachnospiraceae (Fig. S5), including Butyrivibrio hungatei, Anaerostipes hadrus, Butyrivibrio proteoclasticus, Blautia sp. YL58, Herbinix luporum, Blautia hansenii, Roseburia hominis, Lachnoclostridium phocaeense, [Clostridium] bolteae, phytofermentans, Lachnoclostridium Lachnoclostridium sp. YL32 and Pediococcus pentosaceus, was reduced in the intestinal contents of mice fed the HSHF diet (Fig. S5A-D). These Lachnospiraceae members encode a composite inositol catabolism-butyrate biosynthesis pathway, the presence of which is associated with a lower risk of host metabolic disease $^{68}$. Members of the Lachnospiraceae family are among the main producers of short-chain fatty acids. Different taxa of Lachnospiraceae are also associated with different intra- and extraintestinal diseases ${ }^{69}$. Supplement the butyrate-producing Lachnospiraceae is beneficial for the intestinal barrier ${ }^{70}$, probiotic for treating stress-induced visceral hypersensitivity ${ }^{71}$, the butyrate-producing species $R$. inulinivorans includes strains able to grow on inulin and FOS in pure culture ${ }^{72}$. Furuya and colleagues revealed that Blautia hansenii can hydrolyze glucosylceramide to ceramide in plants ${ }^{73}$. Previously, we showed that circNF1-419 could regulate the synthesis and metabolism of ceramides, and the changed ceramides were enriched in three signaling pathways (neurotrophin, sphingolipid, and adipocytokine $)^{41}$. We could conclude that the couples of Blautia and ceramide, butyrate biosynthesis bacteria, and butyrate might be one of interactions between circRNA and microbiome-gut-brain axis, but we still need much more evidence.

Peptostreptococcaceae, a family within the order Clostridiales (Fig. S5), includes the genera Peptostreptococcus, Acetoanaerobium, Proteocatella, Sporacetigenium, Filifactor, and Tepidibacter. The genera Acetoanaerobium, Sporacetigenium and Proteocatella are monospecific. Representatives of the family have different cell morphology, which varies from cocci to rods and filaments. 
Species of Filifactor, Proteocatella, Sporacetigenium, and Tepidibacter form endospores. All members of the family are anaerobes with have a fermentative type of metabolism. The genus Tepidibacter contains moderately thermophilic species. Members of Peptostreptococcaceae are found in different habitats, including the human body, manure, soil, and sediments. Species of Peptostreptococcus and Filifactor are components of the human oral microbiome $^{74}$. Metagenomics sequencing showed that the relative abundance of Peptoclostridium acidaminophilum and Clostridioides difficile was increased in HSHF diet-fed mice. P. acidaminophilum can ferment amino acids ${ }^{75}$. C. difficile is a spore-forming, anaerobic, intestinal pathogen that causes severe diarrhea that can lead to death ${ }^{76} . P$. acidaminophilum and $C$. difficile had a negative correlation with expression of Tpm3 and Dusp6, and a positive correlation with expression of 5-HT and 5-HIAA, but the mechanism of action needs further analysis. Bacteria can produce a range of major neurotransmitters, and substantial evidence has accumulated around the microbiotamediated influence of those compounds ${ }^{13}$. However, the microbiota can also influence levels of neurotransmitters, including histamine, gasotransmitters, neuropeptides, steroids, and endocannabinoids.

\section{Conclusions}

We demonstrated again that consumption of a HSHF diet-induced dysbacteriosis, damaged the intestinal tract, and changed the neurotransmitter metabolism in the intestine and brain. Our new findings were that consumption of a HSHF diet caused changes in brain function and circRNA profiles. Additional experiments found that the GM byproduct of TMAO could degrade some circRNAs, and the basal level of the GM decided the conversion rate of choline to TMAO. A change in the abundance of $C$. albicans and/or K. pneumoniae could influence the cholinergic system. These findings demonstrate a new link between metabolism, brain circRNAs, and the GM, enlarge the microbiome-transcriptome linkage library and provide more information on the gut-brain axis.

\section{Study limitations}

There are several strengths with this study, but there are also limitations. For example, the study lacks a rescue test with large number of single bacteria, so we have not fully identified the one-on-one communication/interaction mechanism between neurotransmitter, circRNA and single bacteria, and therefore could not find the signaliccgeted strains in germ-free mice with multiomics studies to reveal the interaction of neurotransmitter and circRNAs on the microbiome-gut-brain axis, or knock-out mice are needed in the future for positive validations.

\section{Key resources table}

\begin{tabular}{|c|c|c|}
\hline REAGENT or RESOURCE & SOURCE & IDENTIFIER \\
\hline \multicolumn{3}{|l|}{ Antibodies } \\
\hline Anti-IBA-1 & Proteintech & 10904-1-AP \\
\hline Anti-GFAP & Proteintech & 20746-1-AP \\
\hline Anti-AchE & Proteintech & 17975-1-AP \\
\hline Anti-AMP & Proteintech & 13379-1-1P \\
\hline Anti-CHRNA1 & Proteintech & 10613-1AP \\
\hline Anti-CHRNB1 & Proteintech & 11553-1-AP \\
\hline Anti-PPAR- $\gamma$ & Proteintech & 11643-1-AP \\
\hline Anti-TNF-a & Abcam & GR168358-1 \\
\hline Anti-NF-kB p65 & Abcam & 16502 \\
\hline Anti-IL-2 & Proteintech & 60306-1-lg \\
\hline Anti-MAOA & Proteintech & 10539-1-AP \\
\hline Anti-MAOB & Proteintech & 12602-1-AP \\
\hline Anti-COMT & Proteintech & 14754-1-AP \\
\hline Anti-RIG-I & Affinity & DF6107 \\
\hline$\beta$-Actin (13E5) & CST & $4970 \mathrm{~S}$ \\
\hline GAPDH & Abcam & GR3207992-4 \\
\hline
\end{tabular}

\begin{tabular}{lll} 
Chemicals & & \\
\hline Hematoxylin & Servicebio & G1004 \\
Eosin & Servicebio & G1001 \\
Color separation solution & Servicebio & G1039 \\
Diaminobenzidine (DAB) & Servicebio & G1212 \\
Goat anti-rabbit lgG(H+ & Affinity & S0001 \\
L) HRP & & \\
Citrate buffer pH 6.0 & Servicebio & G1202 \\
Anhydrous ethanol & Guangzhou Guanghua Sci- & 1.17113 .023 \\
& Tech cO., Ltd & \\
Penicillin streptomycin & CORNING & 30002304 \\
solution & & \\
Phosphate-buffer saline & CORNING & 19117004 \\
Fetal bovine serum & Gibco & 1932595 \\
Trimethylamine N-Oxide & TOKYO CHEMICAL INDUSTRY & 3 EVVJG-TN \\
anhydrous & CO., LTD & \\
0.25\% Trypsin-EDTA (1X) & Gibco & 2042337 \\
DMEM basic (1x) & Gibco & 8119090 \\
\hline
\end{tabular}




\section{Critical Commercial Assays}

\begin{tabular}{|c|c|c|}
\hline LDL-C Kit & $\begin{array}{l}\text { Nanjing Jiancheng } \\
\text { Bioengineering Institute }\end{array}$ & 20180512 \\
\hline HDL-C Kit & $\begin{array}{l}\text { Nanjing Jiancheng } \\
\text { Bioengineering Institute }\end{array}$ & 20180508 \\
\hline TG Kit & $\begin{array}{l}\text { Nanjing Jiancheng } \\
\text { Bioengineering Institute }\end{array}$ & 20180609 \\
\hline T-CHO Kit & $\begin{array}{l}\text { Nanjing Jiancheng } \\
\text { Bioengineering Institute }\end{array}$ & 20190523 \\
\hline ACH Kit & $\begin{array}{l}\text { Nanjing Jiancheng } \\
\text { Bioengineering Institute }\end{array}$ & 20190505 \\
\hline A-CHE Kit & $\begin{array}{l}\text { Nanjing Jiancheng } \\
\text { Bioengineering Institute }\end{array}$ & 20190318 \\
\hline RNAiso Plus & TaKaRa & 9108 \\
\hline PrimeScript ${ }^{T M} R T$ reagent kit & TaKaRa & RR047A \\
\hline $\begin{array}{l}\text { PrimeScript }{ }^{T M} \text { RT reagent kit } \\
\text { (RT-PCR) }\end{array}$ & TaKaRa & RR037A \\
\hline $\begin{array}{l}\text { TB Green }{ }^{\mathrm{TM}} \text { Premix Ex } \\
\text { Taq }^{\mathrm{TM}} \|\end{array}$ & TaKaRa & RR820A \\
\hline Nissol stain kit & Servicebio & G10020 \\
\hline Silver staining kit & Servicebio & G10021 \\
\hline
\end{tabular}

\section{Deposited Data}

$\begin{array}{lll}\text { RNA sequencing data } & \text { This paper } & \text { PRJNA553830 } \\ \text { circRNA sequencing data } & \text { This paper } & \text { PRJNA553830 }\end{array}$

\section{Software and algorithms}

\begin{tabular}{lll}
\hline GraphPad priam & GraphPad software & N/A \\
Image J & NIH & N/A \\
SIMCA & Umetrics AB & N/A \\
\hline
\end{tabular}

\section{Others}

Choline chloride

Aladdin

C108897

High sugar \& fat diet Beijing HFK Bioscience Co., LTD

(2014)06059

Standard chow Beijing HFK Bioscience Co., LTD

\section{Acknowledgements}

We would like to thank Zhang Maolei for helpful advises in the preparation of this study. Sequencing services were provided by Guangzhou Geneseed Biotech Co., Ltd. Guangzhou, and Biomarker Technologies Co., Ltd. Beijing, China. The present work was supported by the financial support from the National Natural Science Foundation of China (81701086); Guangdong Basic and Applied Basic Research Foundation (2021A1515011338); Guangzhou Health Commission Traditional Chinese Medicine and Integrated Chinese and
Western Medicine Science and Technology Project (20212A011027); GDAS' Project of Science and Technology Development (2019GDASYL- 0104007).

\section{Author details}

'School of Basic Medical Science, Guangzhou University of Chinese Medicine, Guangdong, Guangzhou 510120, China. ${ }^{2}$ State Key Laboratory of Applied Microbiology Southern China; Guangdong Provincial Key Laboratory of Microbial Culture Collection and Application; Guangdong Open Laboratory of Applied Microbiology; Institute of Microbiology, Guangdong Academy of Sciences, Guangzhou 510070, China. ${ }^{3}$ Academy of Life Sciences, Jinan University, Guangdong Province, Guangzhou 510000, China. ${ }^{4}$ Chengdu University of Traditional Chinese Medicine, Chengdu 610075, China. ${ }^{5}$ The Fifth Affiliated Hospital of Guangzhou Medical University, Guangzhou 510700, China

\section{Author contributions}

All the authors designed this study; Y.G., X.Z and M.Z. wrote the manuscript; D.C., Y.G., D.W., X.Y. carried out the computational analyses; D.C., Y.G., L.Q., X.Z. and M.Z. collected animal physiological data and fecal samples, extracted ruminal DNA, did the physiologial and biochemical indexes measurement; X.T., Y.G., and X.Z. did the western bolting analysis; L.Q. and M.Z. did the metabolomic analysis; D.C., Y.G., L.Q., and Shuai O collected data regarding the microbial metabolic networks and transcriptome analysis; M.Z., Y.X. and H.L. helped to design the study and to develop the multi-omics analysis methods, reviewed this manuscript and offer all the necessary research start-up fund, experimental platform. All authors read and approved the final manuscript.

\section{Data availability}

Data collection and sharing for this project was funded by the National Natural Science Foundation of China.

\section{Ethics approval}

The animal protocols used in this work were approved by the Institutional Animal Care and Use committee of the Center of Laboratory Animals of the Guangdong Institute of Microbiology.

\section{Conflict of interest}

The authors declare no competing interests.

\section{Publisher's note}

Springer Nature remains neutral with regard to jurisdictional claims in published maps and institutional affiliations.

Supplementary information The online version contains supplementary material available at https://doi.org/10.1038/s41398-021-01443-2.

Received: 1 December 2020 Revised: 5 May 2021 Accepted: 12 May 2021 Published online: 27 May 2021

\section{References}

1. Chen, $H$. et al. A forward chemical genetic screen reveals gut microbiota metabolites that modulate host physiology. Cell 177, 1217-1231 (2019).

2. Rooks, M. G. \& Garrett, W. S. Gut microbiota, metabolites and host immunity. Nat. Rev. Immunol. 16, 341-352 (2016).

3. Torres-Fuentes, C., Schellekens, H., Dinan, T. G. \& Cryan, J. F. The microbiotagut-brain axis in obesity. Lancet Gastroenterol. Hepatol. 2, 747-756 (2017).

4. Westfall, S. et al. Microbiome, probiotics and neurodegenerative diseases: deciphering the gut brain axis. Cell. Mol. Life Sci. 74, 3769-3787 (2017).

5. Stilling, R. M. et al. The neuropharmacology of butyrate: The bread and butter of the microbiota-gut-brain axis? Neurochem. Int. 99, 110-132 (2016).

6. Reinshagen, M. Neuropods übermitteln Informationen über Nahrungsmittel im Darm über vagale Neuronen in Millisekunden and as Gehirn. Z. f.ür. Gastroenterologie 57, 335 (2019).

7. Liu, P. et al. Altered microbiomes distinguish Alzheimer's disease from amnestic mild cognitive impairment and health in a Chinese cohort. Brain Behav. Immun. 80, 633-643 (2019).

8. Li, B. et al. Mild cognitive impairment has similar alterations as Alzheimer's disease in gut microbiota. Alzheimers Dement 15, 1357-1366 (2019). 
9. van Kessel, S. P. et al. Gut bacterial tyrosine decarboxylases restrict levels of levodopa in the treatment of Parkinson's disease. Nat. Commun. 10, 310 (2019).

10. O'Neill, C. Gut microbes metabolize Parkinson's disease drug. Science $\mathbf{3 6 4}$ 1030-1031 (2019).

11. Fujisaka, S. et al. Diet, genetics, and the gut microbiome drive dynamic changes in plasma metabolites. Cell Rep. 22, 3072-3086 (2018).

12. Thaiss, C. A. et al. Hyperglycemia drives intestinal barrier dysfunction and risk for enteric infection. Science 359, 1376-1383 (2018).

13. Strandwitz, P. Neurotransmitter modulation by the gut microbiota. Brain Res 1693, 128-133 (2018).

14. Olson, C. A. et al. The gut microbiota mediates the anti-seizure effects of the ketogenic diet. Cell 173, 1728-1741 (2018).

15. Strandwitz, P. et al. GABA-modulating bacteria of the human gut microbiota. Nat. Microbiol. 4, 396-403 (2019).

16. Worthy, S. E. et al. Identification of attractive odorants released by preferred bacterial food found in the natural habitats of C. elegans. PLOS ONE 13, e201158 (2018).

17. O Donnell, M. P., Fox, B. W., Chao, P., Schroeder, F. C. \& Sengupta, P. A neurotransmitter produced by gut bacteria modulates host sensory behaviour. Nature 583, 415-420 (2020).

18. Jiang, C., Li, G., Huang, P., Liu, Z. \& Zhao, B. The gut microbiota and Alzheimer's disease. J. Alzheimers Dis. 58, 1-15 (2017).

19. Kaelberer, M. M. et al. A gut-brain neural circuit for nutrient sensory transduction. Science 361, t5236 (2018).

20. Zheng, $P$. et al. The gut microbiome from patients with schizophrenia modulates the glutamate-glutamine-GABA cycle and schizophrenia-relevant behaviors in mice. Sci. Adv. 5, u8317 (2019).

21. Liu, R. et al. Gut microbiome and serum metabolome alterations in obesity and after weight-loss intervention. Nat. Med. 23, 859-868 (2017).

22. Saffouri, G. B. et al. Small intestinal microbial dysbiosis underlies symptoms associated with functional gastrointestinal disorders. Nat. Commun. 10, 2012 (2019).

23. Jeong, J. H., Lee, D. K. \& Jo, Y. Cholinergic neurons in the dorsomedial hypothalamus regulate food intake. Mol. Metab. 6, 306-312 (2017).

24. Hampel, $H$. et al. The cholinergic system in the pathophysiology and treatment of Alzheimer's disease. Brain 141, 1917-1933 (2018).

25. Kwon, Y. H. et al. Modulation of gut microbiota composition by serotonin signaling influences intestinal immune response and susceptibility to colitis. Cell. Mol. Gastroenterol. Hepatol. 7, 709-728 (2019).

26. Cox, M. A. et al. Choline acetyltransferase-expressing $T$ cells are required to control chronic viral infection. Science 363, 639-644 (2019).

27. Zhu, W. et al. Gut microbial metabolite TMAO enhances platelet hyperreactivity and thrombosis risk. Cell 165, 111-124 (2016).

28. Vogt, N. M. et al. The gut microbiota-derived metabolite trimethylamine Noxide is elevated in Alzheimer's disease. Alzheimers Res. Ther. 10, 124 (2018).

29. Pecoraro, V. et al. A subnanomolar concentration of Pituitary Adenylate Cyclase-Activating Polypeptide (PACAP) pre-synaptically modulates glutamatergic transmission in the rat hippocampus acting through acetylcholine. Neuroscience 340, 551-562 (2017).

30. Subramaniam, S. \& Fletcher, C. Trimethylamine N-oxide: breathe new life. Br. J. Pharm. 175, 1344-1353 (2018).

31. Doenyas, C. Gut microbiota, inflammation, and probiotics on neural development in autism spectrum disorder. Neuroscience 374, 271-286 (2018).

32. Lach, G., Schellekens, H., Dinan, T. G. \& Cryan, J. F. Anxiety, depression, and the microbiome: a role for gut peptides. Neurotherapeutics 15, 36-59 (2018).

33. $\mathrm{Xu}, \mathrm{R}$. et al. Altered gut microbiota and mucosal immunity in patients with schizophrenia. Brain, Behav., Immun. 85, 120-127 (2020).

34. Seitz, J. et al. The impact of starvation on the microbiome and gut-brain interaction in anorexia nervosa. Front. Endocrinol. 10, 41 (2019).

35. Camara-Lemarroy, C. R., Metz, L., Meddings, J. B., Sharkey, K. A. \& Wee, Y. V. The intestinal barrier in multiple sclerosis: implications for pathophysiology and therapeutics. Brain 141, 1900-1916 (2018).

36. Dahlin, M. \& Prast-Nielsen, S. The gut microbiome and epilepsy. EBioMedicine 44, 741-746 (2019).

37. Sampson, T. R. et al. Gut microbiota regulate motor deficits and neuroinflammation in a model of Parkinson's disease. Cell 167, 1469-1480 (2016).

38. Memczak, S. et al. Circular RNAs are a large class of animal RNAs with regulatory potency. Nature 495, 333-338 (2013).

39. Weigelt, C. M. et al. An insulin-sensitive circular RNA that regulates lifespan in drosophila. Mol. Cell. 79, 268-279 (2020).
40. Hentze, M. W. \& Preiss, T. Circular RNAs: splicing's enigma variations. EMBO J. 32, 923-925 (2013).

41. Diling, $C$. et al. CircNF1-419 improves the gut microbiome structure and function in AD-like mice. Aging (Albany NY) 12, 260-287 (2020).

42. Tong, J. et al. Brain monoamine oxidase $B$ and $A$ in human parkinsonian dopamine deficiency disorders. Brain 140, 2460-2474 (2017).

43. Sogodogo, E., Drancourt, M. \& Grine, G. Methanogens as emerging pathogens in anaerobic abscesses. Eur. J. Clin. Microbiol Infect. Dis. 38, 811-818 (2019).

44. Drancourt, M. et al. Evidence of archaeal methanogens in brain abscess. Clin. Infect. Dis. 65, 1-5 (2017).

45. Wilder-Smith, C. H., Olesen, S. S., Materna, A. \& Drewes, A. M. Breath methane concentrations and markers of obesity in patients with functional gastrointestinal disorders. U. Eur. Gastroenterol. J. 6, 595-603 (2018).

46. Zhang, B., Gao, M., Shen, J. \& He, D. Inhaled methane protects rats against neurological dysfunction induced by cerebral ischemia and reperfusion injury: PI3K/Akt/HO-1 pathway involved. Arch. Med. Res. 48, 520-525 (2017).

47. Pimentel, M. \& Lembo, A. Microbiome and its role in irritable bowel syndrome. Dig. Dis. Sci. 65, 829-839 (2020).

48. Yamada, T. et al. Expression of acetylcholine (ACh) and ACh-synthesizing activity in Archaea. Life Sci. 77, 1935-1944 (2005).

49. Zhang, D. et al. The mycobiota of the human body: a spark can start a prairie fire. Gut Microbes 11, 655-679 (2020).

50. Schei, K. et al. Early gut mycobiota and mother-offspring transfer. Microbiome 5, 107 (2017).

51. Li, X. V., Leonardi, I. \& Iliev, I. D. Gut mycobiota in immunity and inflammatory disease. Immunity 50, 1365-1379 (2019).

52. Richard, M. L. \& Sokol, H. The gut mycobiota: insights into analysis, environmental interactions and role in gastrointestinal diseases. Nat. Rev. Gastroenterol. Hepatol. 16, 331-345 (2019).

53. Bacher, P. et al. Human anti-fungal Th17 immunity and pathology rely on cross-reactivity against Candida albicans. Cell 176, 1340-1355 (2019).

54. Valentine, M., Benade, E., Mouton, M., Khan, W. \& Botha, A. Binary interactions between the yeast Candida albicans and two gut-associated Bacteroides species. Micro. Pathog. 135, 103619 (2019).

55. Witchley, J. N. et al. Candida albicans morphogenesis programs control the balance between gut commensalism and invasive infection. Cell Host Microbe 25, 432-443 (2019).

56. Nile, C. et al. Repurposing pilocarpine hydrochloride for treatment of Candida albicans infections. mSphere 4, e00689-18 (2019).

57. Haase, S., Haghikia, A., Wilck, N., Muller, D. N. \& Linker, R. A. Impacts of microbiome metabolites on immune regulation and autoimmunity. Immunology 154, 230-238 (2018).

58. Sherwin, E., Bordenstein, S. R., Quinn, J. L., Dinan, T. G. \& Cryan, J. F. Microbiota and the social brain. Science $\mathbf{3 6 6}$, eaar2016 (2019).

59. Xu, Z. et al. Chronic alcohol exposure induced gut microbiota dysbiosis and its correlations with neuropsychic behaviors and brain BDNF/Gabra1 changes in mice. Biofactors 45, 187-199 (2019).

60. Ribas, D. et al. The acetate uptake transporter family motif "NPAPLGL(M/S)" is essential for substrate uptake. Fungal Genet. Biol. 122, 1-10 (2019).

61. Wolfson, R. L. et al. Sestrin2 is a leucine sensor for the mTORC1 pathway. Science 351, 43-48 (2016).

62. Yang, $\mathrm{H}$. et al. Mechanisms of mTORC1 activation by RHEB and inhibition by PRAS40. Nature 552, 368-373 (2017).

63. latsenko, I., Boquete, J. P. \& Lemaitre, B. Microbiota-derived lactate activates production of reactive oxygen species by the intestinal NADPH oxidase nox and shortens Drosophila lifespan. Immunity 49, 929-942 (2018).

64. Liu, C. X. et al. Structure and degradation of circular RNAs regulate PKR activation in innate immunity. Cell 177, 865-880 (2019).

65. Eimer, W. A. et al. Alzheimer's disease-associated beta-amyloid is rapidly seeded by herpesviridae to protect against brain infection. Neuron $\mathbf{9 9}$ 56-63 (2018).

66. Dhar, D. \& Mohanty, A. Gut microbiota and Covid-19- possible link and implications. Virus Res 285, 198018 (2020).

67. Shkoporov, A. N. et al. The human gut virome is highly diverse, stable, and individual specific. Cell Host Microbe 26, 527-541 (2019).

68. Zeng, $X$. et al. Higher risk of stroke is correlated with increased opportunistic pathogen load and reduced levels of butyrate-producing bacteria in the gut. Front Cell Infect. Microbiol 9, 4 (2019).

69. Vacca, M. et al. The controversial role of human gut Lachnospiraceae. Microorganisms 8, 573 (2020) 
70. Sasaki, K. et al. Construction of a model culture system of human colonic microbiota to detect decreasedlachnospiraceae abundance and butyrogenesis in the feces of ulcerative colitis patients. Biotechnol. J. 14, 1800555 (2019).

71. Zhang, J. et al. Beneficial effect of butyrate-producing Lachnospiraceae on stress-induced visceral hypersensitivity in rats. J. Gastroenterol. Hepatol. 34, 1368-1376 (2019).

72. Louis, P., Young, P., Holtrop, G. \& Flint, H. J. Diversity of human colonic butyrate-producing bacteria revealed by analysis of the butyryl-CoA:acetate CoA-transferase gene. Environ. Microbiol 12, 304-314 (2010).

73. Furuya, H., Ide, Y., Hamamoto, M., Asanuma, N. \& Hino, T. Isolation of a novel bacterium, Blautia glucerasei sp. nov., hydrolyzing plant glucosylceramide to ceramide. Arch. Microbiol. 192, 365-372 (2010).
74. Galperin, M. Y., Brover, V., Tolstoy, I. \& Yutin, N. Phylogenomic analysis of the family Peptostreptococcaceae (Clostridium cluster XI) and proposal for reclassification of Clostridium litorale (Fendrich et al. 1991) and Eubacterium acidaminophilum (Zindel et al. 1989) as Peptoclostridium litorale gen. nov. comb. nov. and Peptoclostridium acidaminophilum comb. nov. Int. J. Syst. Evol. Micr 66, 5506-5513 (2016).

75. Mei, R., Nobu, M. K. \& Liu, W. T. Identifying anaerobic amino acids degraders through the comparison of short-term and long-term enrichments. Env. Microbiol. Rep. 12, 173-184 (2020).

76. Sandhu, B. K. \& McBride, S. M. Clostridioides difficile. Trends Microbio/ 26 1049-1050 (2018). 